\title{
On the Covariant Structure of the Two-Point Function
}

\author{
A. I. OKSAK \\ Joint Institute for Nuclear Research, Dubna, USSR \\ and \\ I. T. TODOROV* \\ Institute for Advanced Study, Princeton, New Jersey
}

\begin{abstract}
The spectral representation of the two-point function for arbitrary fields proposed recently [1] is rigorously proved and analyzed. The problem is treated in momentum space where the covariant structure is simpler because of the spectrum conditions. For finite-component fields the explicit "matrix" structure is found in coordinate space too and is applied to the definition of time-ordered Green functions for arbitrary spin. The decomposition of the two-point function into kernels of definite spin is carried out in the general case, a necessary and sufficient condition for the growth of the coefficients in this decomposition being given. The positive-definiteness condition (in the case of Hermitian conjugate fields) is fulfilled automatically by the elementary kernels.

The formalism of homogeneous distributions in two dimensional complex domain [2] is used throughout the paper.
\end{abstract}

\section{Contents}

1. Introduction . . . . . . . . . . . . . . . . . . . . . . . 272

2. General Form of the Invariant Two-Point Function in Momentum Space . . . 274

2.1. Differential Form of the Invariance Condition . . . . . . . . . . . . . . 274

2.2. Any Invariant Distribution in $D^{\prime}\left(V_{+} \times \tilde{C}_{2} \times \tilde{C}_{2}\right)$ is a "Function" of the Algebraic Invariants . . . . . . . . . . . . . . . . . . . . . . . . . . 275

2.3. Irreducibility Condition. Homogeneous Invariant Distributions . . . . . . . 278

3. The Two-Point Function for Finite-Component Fields . . . . . . . . . . . 281

3.1. The Invariant Distribution $F(x ; \zeta)$. . . . . . . . . . . . . . . . . . . . . 281

3.2. Covariant Structure of the Two-Point Function for Finite Dimensional Representation of $S L(2, C)$. . . . . . . . . . . . . . . . . . . . . . . . . . 283

3.3. Application to the Definition of the Time-Ordered Green's Function for Arbitrary Spinor Fields . . . . . . . . . . . . . . . . . . . . . . . . . . 284

4. Decomposition of the Two-Point Function with Respect to Spin . . . . . . . 287

4.1. Elementary Invariant Kernels and Poincaré Decomposition of the Two-Point Function . . . . . . . . . . . . . . . . . . . . . . . . . . . . . . . 287

4.2. Remark on the Positive-Definiteness Condition . . . . . . . . . . . . . . . 290 Appendix A. Isomorphism between $\mathfrak{D}_{\chi}^{\prime}$ and the Subspace of Homogeneous Distribution of Index $-\chi$ of $D^{\prime}\left(\tilde{C}_{2}\right)$. . . . . . . . . . . . . . . . . . . . . . . . . . 291

A.1. Synopsis on the Description of the Irreducible Representations of $S L(2, C)$ in Terms of Homogeneous Functions ([2]). . . . . . . . . . . . . . . . . . 291

${ }^{\star}$ On leave of absence from Joint Institute for Nuclear Research, Dubna, USSR and from Physical Institute of the Bulgarian Academy of Sciences, Sofia, Bulgaria.

20 Commun. math. Phys., Vol. 14 
A.2. The Mapping of $D\left(\tilde{C}_{2}\right)$ on $\mathfrak{D}_{\chi}$ and its Adjoint . . . . . . . . . . . . . . . . 292

Appendix B. Regularity of the Mapping $O_{1} \rightarrow \Omega_{1}$ (Proof of Lemma 2.1). . . . . . . 294

Appendix C. General Form of Covariant Tensor-Valued Distributions (Proof of

Theorem 3.1) . . . . . . . . . . . . . . . . . . . . . . . . . . . . . . . 295

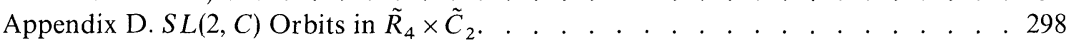

Appendix E. Expansion of Generalized Functions of $\mathscr{D}^{\prime}([-1,1])$ in Jacobi Poly-

nomials . . . . . . . . . . . . . . . 301

References . . . . . . . . . . . . . . . . . . . . 304

\section{Introduction}

A representation was proposed recently [1] for the invariant twopoint function in a theory involving infinite-component fields. The present paper is devoted to a rigorous derivation and further analysis of this representation.

We start with the precise formulation of the problem and give a summary of results.

Let $\tau$ be a linear representation $A \rightarrow T(A)$ of the quantum mechanical Lorentz group $S L(2, C)$ where $T(A)$ are (continuous) operators in a topological vector space $\mathfrak{D}$. Let further $S\left(R_{4}\right)$ be the Schwartz space of infinitely smooth fast decreasing functions in four dimensions. A relativistic quantized field $\psi(u ; f)\left(u \in S\left(R_{4}\right), f \in \mathfrak{D}\right)$ is defined as a bilinear weakly continuous mapping of $S\left(R_{4}\right) \times \mathfrak{D}$ into the set of (unbounded) operators with a common invariant dense domain $\Omega$ in the Hilbert space $\mathfrak{H}$ of state vectors (cf. [3]).

We assume that a unitary representation $U(a, A)$ of the covering of the Poincare group $\operatorname{ISL}(2, C)$ is acting in $\mathfrak{H}$, satisfying the usual requirements [3] (including the existence and uniqueness of the invariant vacuum state $|0\rangle \in \Omega$ ). The transformation law for the field is given by

$$
U(a, A) \psi(u ; f) U^{-1}(a, A)=\psi\left(u_{\{a, A\}} ; T(A) f\right)
$$

where $u_{\{a, A\}}(x)=u\left(\Lambda^{-1}(x-a)\right)$, and $\Lambda=\Lambda(A)$ is the proper Lorentz transformation defined by

$$
A \sigma_{\mu} A^{*}=\sigma_{v} \Lambda^{v} \mu
$$

$\left(\sigma_{j}, j=1,2,3\right.$ are the Pauli matrices, $\sigma_{0}$ is the $2 \times 2$ unit matrix).

In view of the translation invariance the two-point function (i.e. the vacuum expectation value of the product of two fields) can be written in the form

$F_{\varphi \psi}(x-y ; f, g)=\langle 0|\varphi(x ; f) \psi(y ; g)| 0\rangle=\int K_{\varphi \psi}(p ; f, g) e^{-i p(x-y)} d^{4} p$.

The tempered distribution $K_{\varphi \psi}$ is called spectral function. To avoid unnecessary complications we assume the strong form of spectrum 
condition with a mass gap $[3]^{1}$ ) which implies that

$$
\operatorname{supp} K(p ; f ; g) \subset V_{+}=\left\{p\left|p^{0}>\right| \boldsymbol{p} \mid\right\} .
$$

We shall use here the notation ${ }^{2} \chi=[k, c]$ for the irreducible representations of $S L(2, C)$ where $k$ is an integer or half-integer and $c$ is in general an arbitrary complex number (for the principal series of unitary representations $c$ is pure imaginary [4]).

Let the fields $\varphi(x ; f)$ and $\psi(x ; g)$ transform under the irreducible representations $\chi_{1}$ and $\chi_{2}$ of $S L(2, C)$, respectively; then we suppose that $f \in \mathfrak{D}_{-\chi_{1}}$ and $g \in \mathfrak{D}_{-\chi_{2}}$ transform respectively under $T_{-\chi_{1}}$ and $T_{-\chi_{2}}$. Our main result is the derivation of the general form of the spectral function $K(u ; f ; g)$ considered as a trilinear functional on ${ }^{3} D\left(V_{+}\right) \times \mathfrak{D}_{-\chi_{1}} \times \mathfrak{D}_{-\chi_{2}}$ satisfying the invariance condition

$$
K\left(u_{\{0, A\}} ; T_{-\chi_{1}}(A) f ; T_{-\chi_{2}}(A) g\right)=K(u ; f ; g) .
$$

The solution of this problem is based on the isomorphism (established in Appendix A) between the space $\mathfrak{D}_{-\chi}^{\prime}$ of linear functionals on $\mathfrak{D}_{-\chi}$ and the space $d_{\chi}$ of homogeneous distributions of index $\chi$ in the two-dimensional complex domain $\tilde{C}_{2}=C_{2} \backslash\{0\}$. This isomorphism considerably simplifies the surch of polylinear Lorentz-invariant forms by means of distribution technique. The general invariant kernel $K(p ; z ; w)$ $\in D^{\prime}\left(V_{+} \times \tilde{C}_{2} \times \tilde{C}_{2}\right)$ satisfying conditions (2.2), (2.3) is given by Theorem 2.2. It is non vanishin only for integer $k_{1}-k_{2}$ and is characterized by a generalized function $g(\tau, v) \in \mathscr{D}^{\prime}\left(R_{+}^{1} \times[-1,1]\right)$ which depends on two invariants

$\tau=p^{2} \quad$ and $\quad v=\frac{|z \tilde{p} \bar{w}|^{2}-p^{2}|z \varepsilon w|^{2}}{z \tilde{p} \bar{z} \cdot w \tilde{p} \bar{w}}\left(\varepsilon=\left(\begin{array}{rr}0 & 1 \\ -1 & 0\end{array}\right), \tilde{p}=p^{\mu} \sigma_{\mu}\right)$.

[Here $R_{1}^{+}$is the (open) positive semi-axis: $0<\tau<\infty$.]

The case of finite-component fields is studied in more detail in Section 3. The analysis of Methée $[6,7]$ is extended to the case of tensor

${ }^{1}$ If the spectrum condition is not fulfilled then one should expect a more complicated structure for the functional $K(u ; f ; g)$. This is suggested in particular by the structure of the $S L(2, C)$ orbits in the space of variables of the kernel $K(p ; z, w)$ (see Appendix D).

${ }^{2}$ The pair $\chi=\left(n_{1}, n_{2}\right)$ used in [2] is related to [k,c] by $n_{1}=c+k, n_{2}=c-k$. We shall realize $T_{\chi}$ as a set of argument transformations in the space $\mathfrak{D}_{\chi}$ of infinitely differentiable homogeneous functions of two complex variables (see Appendix A). We shall make use of the fact that the representation $T_{\chi}^{\prime}$ (acting in $\mathcal{D}_{\chi}^{\prime}$ ) is an extension of $T_{\chi}: T_{\chi}^{\prime} \supset T_{\chi}$ (see [2]).

${ }^{3}$ From the assumed temperateness of the fields it follows that the functional $K(u ; f ; g)$ can be extended to the larger space $S\left(V_{+}\right) \times \mathfrak{D}_{-\chi_{1}} \times \mathfrak{D}_{-\chi_{2}}$. Moreover, translation invariance implies that it is a measure with respect to $p$. However, no additional difficulties appear when treating the problem in the more general formulation given in the text which incorporates for instance the Jaffe fields [5]. 
Lorentz-covariant distributions $F(x)$. Such distributions are represented as finite sums of covariant polynomials of $x$ (or $\partial / \partial x$ ) multiplied by $L_{+}^{\uparrow}-$ invariant scalar distributions (in analogy with the case of analytic tensor functions treated in [8], Section 4). This result allows us to reduce the problem of defining the Green tensor functions in terms of Wightman functions to the corresponding solved problem [9] for scalar fields.

Section 4 is devoted to decomposition of the two-point function with respect to spin. The problem is reduced to the expansion of a generalized function in the interval $[-1,1]$ in Jacobi polynomials. It is found (Appendix E) that a necessary and sufficient condition for such an expansion to make sense is the polynomial boundedness of the coefficients (with respect to their index). For a pair of Hermitian conjugate fields the kernels $K_{s}$ of definite spin are positive-definite.

\section{General Form of the Invariant Two-Point Function in Momentum Space}

\subsection{Differential Form of the Invariance Condition}

Because of the isomorphism between the space $\mathfrak{D}_{-\chi}^{\prime}\left(\right.$ dual to $\mathfrak{D}_{-\chi}$ ) and the space $d_{\chi}$ of homogeneous distributions on $D\left(\tilde{C}_{2}\right)$ (see Appendix A) the set of (continuous) trilinear functionals $K(u ; f, g)$ on $D\left(V_{+}\right)$ $\times \mathfrak{D}_{-\chi_{1}} \times \mathfrak{D}_{-\chi_{2}}$ is isomorphic to the set of homogeneous trilinear functionals $K(u ; F, G)$ on $D\left(V_{+}\right) \times D\left(\tilde{C}_{2}\right) \times D\left(\tilde{C}_{2}\right)$ of indices of homogeneity $\chi_{1}, \chi_{2}$. In accordance with Schwartz nuclear theorem we can describe the functional $K(u ; F, G)$ by its kernel

$$
K(p ; z, w) \in D^{\prime}\left(V_{+} \times \tilde{C}_{2} \times \tilde{C}_{2}\right)
$$

which satisfies the homogeneity condition

$$
K\left(p ; \sqrt{\varrho_{1}} e^{i \frac{\alpha_{1}}{2}} z, \sqrt{\varrho_{2}} e^{i \frac{\alpha_{2}}{2} w}\right)=\varrho_{1}^{c_{1}-1} \varrho_{2}^{c_{2}-1} e^{i\left(k_{1} \alpha_{1}+k_{2} \alpha_{2}\right)} K(p ; z, w)
$$

$\left(\varrho_{j}>0, \chi_{j}=\left[k_{j}, c_{j}\right]\right)$, and is Lorentz invariant.

$$
K\left(\Lambda(A) p ; z A^{-1}, w A^{-1}\right)=K(p ; z, w)
$$

where $A \in S L(2, C), z A \equiv{ }^{t} A z,{ }^{t} A$ is the transposed of $A$, and $\Lambda(A)$ is defined by (1.2).

In order to rewrite (2.3) in an infinitesimal form it is convenient to introduce instead of the four vector $p$ the Hermitian matrix $\tilde{p}$ (cf. (1.5)):

$$
\tilde{p}=p^{\mu} \sigma_{\mu}=\left(\begin{array}{ll}
p^{0}+p^{3} & p^{1}-i p^{2} \\
p^{1}+i p^{2} & p^{0}-p^{3}
\end{array}\right)=\left(p^{a \dot{b}}\right), a, \quad \dot{b}=1,2 .
$$


Eq. (1.2) implies that

$$
\widetilde{\Lambda(A) p}=A \tilde{p} A^{*} .
$$

In terms of $\tilde{p}$ the forward cone $V_{+}$can be defined by $V_{+}=\left\{\tilde{p} \mid p^{11}>0\right.$, $\left.\operatorname{det} \tilde{p}=p^{2}>0\right\}$. We parametrize the complex matrix

$$
A=\left(\begin{array}{ll}
a_{1}^{1} & a_{2}^{1} \\
a_{1}^{2} & a_{2}^{2}
\end{array}\right), a_{1}^{1} a_{2}^{2}-a_{2}^{1} a_{1}^{2}=1
$$

in the neighborhood of the unit element $A=I$ of $S L(2, C)$ by the parameters $a_{1}^{1}, a_{2}^{1}$ and $a_{1}^{2}$. Differentiating (2.3) with respect to these parameters and their complex conjugates in the point $A=I$ we obtain 6 differential equations for $K(p ; z, w)$. We shall write down only four of them (those obtained by differentiation with respect to $a_{2}^{1}, a_{1}^{2}, \bar{a}_{2}^{1}, \bar{a}_{1}^{2}$ ), because they already contain complete information about the Lorentz invariance of $K$ :

$$
\begin{aligned}
& \left(p^{a \dot{c}} \frac{\partial}{\partial p^{b \dot{c}}}-z_{b} \frac{\partial}{\partial z_{a}}-w_{b} \frac{\partial}{\partial w_{a}}\right) \quad K=0, \quad a=1,2, \quad b=3-a ; \\
& \left(p^{c \dot{a}} \frac{\partial}{\partial p^{c \dot{b}}}-\bar{z}_{\dot{b}} \frac{\partial}{\partial \bar{z}_{\dot{a}}}-\bar{w}_{\dot{b}} \frac{\partial}{\partial \bar{w}_{\dot{a}}}\right) \quad K=0, \quad \dot{a}=1,2, \quad \dot{b}=3-\dot{a}
\end{aligned}
$$

(summation is to be carried out over the repeated index $\dot{c}$ (or $c$ ) from 1 to 2$)$.

\subsection{Any Invariant Distribution of $D^{\prime}\left(V_{+} \times \tilde{C}_{2} \times \tilde{C}_{2}\right)$ is a "Function" of the Algebraic Invariants}

We shall find first the general solution of (2.6), (2.7) without using the homogeneity condition. Thus, the result will be valid also for a class of reducible representations of $S L(2, C)$.

We choose the following set of independent algebraic invariants of the transformation group $(\tilde{p}, z, w) \rightarrow\left(A \tilde{p} A^{*}, z A^{-1}, w A^{-1}\right)$ :

$$
\begin{aligned}
& \tau=p^{2} \equiv \operatorname{det} \tilde{p}, \quad v=w \tilde{p} \bar{w}, \\
& s=\sqrt{\tau} z \varepsilon w \equiv \sqrt{p^{2}}\left(z_{1} w_{2}-z_{2} w_{1}\right), \quad t=z \tilde{p} \bar{w} .
\end{aligned}
$$

The image of the domain $V_{+} \times \tilde{C}_{2} \times \tilde{C}_{2}$ of the variables $(\tilde{p}, z, w)$ in the set of invariants $(\tau, v, s, t)$ is $R_{1}^{+} \times R_{1}^{+} \times \tilde{C}_{2}$. This is a consequence of the positive definiteness of $\tilde{p}$ and of the identity

$$
u \equiv z \tilde{p} \bar{z}=\frac{1}{v}\left(|s|^{2}+|t|^{2}\right) .
$$

In order to introduce a regular change of variables in $K(p, z, w)$ we consider the set $V_{+} \times \tilde{C}_{2} \times \tilde{C}_{2}$ as the union of the overlapping domains 
$O_{1}$, in which $w_{2} \neq 0$ and $O_{2}$, in which $w_{1} \neq 0$. Let $\mathfrak{I}_{1}$ be the mapping $(\tilde{p}, z, w) \rightarrow\left(\tau ; v ; s, t ; \lambda_{1}\right), \lambda_{1} \equiv\left(p^{11}, p^{21} ; \eta=w_{1}+\frac{p^{21}}{p^{11}} w_{2}, \varphi=\arg w_{2}\right)$

of $O_{1}$ on $\Omega_{1}=\left\{\tau \in R^{+}, v \in R_{1}^{+},(s, t) \in \tilde{C}_{2}, p^{11} \in R_{1}^{+}, p^{21} \in C_{1},\left|p^{21}\right|<\sqrt{\tau}\right.$; $\left.\eta \in C_{1}, \phi \in R_{1}\right\}\left(\arg w_{2}\right.$ being a multivalued function of $w_{2}$ ). Analogously, we define the mapping $\mathfrak{I}_{2}$

$(\tilde{p}, z, w) \rightarrow\left(\tau ; v ; s, t ; \lambda_{2}\right), \lambda_{2} \equiv\left(p^{22}, p^{12} ; \eta^{\prime}=w_{2}+\frac{p^{12}}{p^{22}} w_{1}, \varphi^{\prime}=\arg w_{1}\right)$

of $\mathrm{O}_{2}$ on a corresponding domain $\Omega_{2}$.

Lemma 2.1. There exists an isomorphism

$$
K(p ; z ; w)=K_{j}\left(\tau ; v ; s, t ; \lambda_{j}\right) \quad j=1,2
$$

between $D^{\prime}\left(O_{j}\right)$ and the space $D^{\prime}\left(\Omega_{j}\right)$ of distributions periodic with respect to $\varphi\left(\right.$ or $\left.\varphi^{\prime}\right)$ of period $2 \pi$.

The proof of this Lemma is given in Appendix B.

Our next step will be to prove, using the invariance conditions (2.6), (2.7), that $K_{j}$ do not depend actually on $\lambda_{j}$. First, we observe that it is sufficient to differentiate $K_{j}$ with respect to the set of variables $\lambda_{j}$, because any "function" of the invariants (2.8) satisfies Eqs. (2.6) and (2.7) identically. We consider in detail only the case $j=1$; the case $j=2$ can be treated analogously.

Inserting (2.11) (for $j=1$ ) into (2.6) for $a=1$ and into (2.7) for $\dot{a}=1$ we find

$$
\begin{aligned}
& p^{11}\left(\frac{\partial K_{1}}{\partial p^{21}}+\frac{\partial K_{1}}{\partial \eta} \frac{w_{2}}{p^{11}}\right)-\frac{\partial K_{1}}{\partial \eta} w_{2}=0, \\
& p^{11}\left(\frac{\partial K_{1}}{\partial p^{12}}+\frac{\partial K_{1}}{\partial \bar{\eta}} \frac{\bar{w}_{2}}{p^{11}}\right)-\frac{\partial K_{1}}{\partial \bar{\eta}} \bar{w}_{2}=0 ;
\end{aligned}
$$

hence

$$
\left.\frac{\partial K_{1}}{\partial p^{21}}=\frac{\partial K_{1}}{\partial p^{21}}=0 \quad \overline{\left(p^{21}\right.}=p^{12}\right) .
$$

Substituting (2.11) in (2.6) for $a=2$ and taking into account (2.12) we obtain

$$
\begin{aligned}
p^{21}( & \left.\frac{\partial K_{1}}{\partial p^{11}}-\frac{\partial K_{1}}{\partial \eta} \frac{p^{21}}{\left(p^{11}\right)^{2}} w_{2}-\frac{\partial K_{1}}{\partial \bar{\eta}} \frac{p^{12}}{\left(p^{11}\right)^{2}} \bar{w}_{2}\right) \\
& -\left(\eta-\frac{p^{21}}{p^{11}} w_{2}\right)\left(\frac{\partial K_{1}}{\partial \eta} \frac{p^{21}}{p^{11}}+\frac{\partial K_{1}}{\partial \varphi} \frac{1}{2 i w_{2}}\right)=0 .
\end{aligned}
$$


Since $K_{1}$ does not depend on $p^{21}, \overline{p^{21}}\left(=p^{12}\right)$ (because of (2.12)) the coefficients to any power of $p^{21}$ and $\overline{p^{21}}$ in (2.13) should vanish. Taking into account that in the domain under consideration $p^{11}>0$ and $w_{2} \neq 0$ we get

$\eta \frac{\partial K_{1}}{\partial \varphi}=0, \quad \frac{\partial K_{1}}{\partial \bar{\eta}}=0, \quad \frac{\partial K_{1}}{\partial p^{11}}-\frac{\eta}{p^{11}} \frac{\partial K_{1}}{\partial \eta}-\frac{i}{2 p^{11}} \frac{\partial K_{1}}{\partial \varphi}=0$.

Analogously, using (2.7) for $\dot{a}=2$ we find

$\bar{\eta} \frac{\partial K_{1}}{\partial \varphi}=0, \quad \frac{\partial K_{1}}{\partial \eta}=0, \quad \frac{\partial K_{1}}{\partial p^{11}}-\frac{\bar{\eta}}{p^{11}} \frac{\partial K_{1}}{\partial \bar{\eta}}+\frac{i}{2 p^{11}} \frac{\partial K_{1}}{\partial \varphi}=0$.

From (2.14) and (2.12) we obtain the desired result:

$$
\frac{\partial K_{1}}{\partial p^{11}}=\frac{\partial K_{1}}{\partial p^{21}}=\frac{\partial K_{1}}{\partial \bar{p}^{21}}=\frac{\partial K_{1}}{\partial \eta}=\frac{\partial K_{1}}{\partial \bar{\eta}}=\frac{\partial K_{1}}{\partial \varphi}=0 .
$$

As far as the domain $\Omega_{1}$ is convex with respect to the variables $\lambda_{1}$ (for fixed $\tau, v, s, t)$ we conclude that there is a distribution $\mathscr{K}_{1}(\tau ; v ; s, t)$ $\in D^{\prime}\left(R_{1}^{+} \times R_{1}^{+} \times \tilde{C}_{2}\right)$ such that

$$
K_{1}\left(\tau, v ; s, t ; p^{11}, p^{21}, p^{12}, \eta, \varphi\right)=\mathscr{K}_{1}(\tau ; v ; s, t) \text { in } \Omega_{1}
$$

or equivalently $K(p ; z, w)=\mathscr{K}_{1}(\tau ; v ; s, t)$ in $O_{1}$.

An analogous reasoning shows that there exists a function of the invariants $\mathscr{K}_{2}(\tau ; v ; s, t) \in D^{\prime}\left(R_{1}^{+} \times R_{1}^{+} \times \tilde{C}_{2}\right)$ which coincides with $K(p ; z, w)$ in $O_{2}$. On the other hand each of the distributions $\mathscr{K}_{j}(j=1,2)$ is uniquely determined by the "values" of $K(p ; z, w)$ in the intersection $O_{1} \cap O_{2}$ (because the image of $O_{1} \cap O_{2}$ in the mapping (2.8) $(p, z, w) \rightarrow(\tau, v, s, t)$ is the whole domain $R_{1}^{+} \times R_{1}^{+} \times \tilde{C}_{2}$ ). Hence $\mathscr{K}_{1}=\mathscr{K}_{2}=\mathscr{K}$ and we have proved the following theorem.

Theorem 2.1. Any distribution $K(p ; z, w) \in D^{\prime}\left(V_{+} \times \tilde{C}_{2} \times \tilde{C}_{2}\right)$ satisfying the invariance condition (2.3) can be written in the form

$$
K(p ; z, w)=\mathscr{K}(\tau ; v ; s, t)
$$

where $\tau, v, s, t$ are the invariants (2.8) and $\mathscr{K} \in D^{\prime}\left(R_{1}^{+} \times R_{1}^{+} \times \tilde{C}_{2}\right)$.

Remark. We have not used symmetric variables with respect to $z$ and $w: t, v(2.8), u(2.9)$ and $\sigma=\frac{s}{\sqrt{\tau}}=z \varepsilon w$, because the change of variables $(\tau, v, s, t) \rightarrow(u, v, \sigma, t)$ is singular (for $s=0$ its Jacobian vanishes). 


\subsection{Irreducibility Condition. Homogeneous Invariant Distributions}

In terms of the kernel $\mathscr{K}(\tau ; v ; s, \mathrm{t})(2.16)$ the homogeneity condition assumes the form

$$
\begin{gathered}
\mathscr{K}\left(\tau ; \varrho_{2} v ; \sqrt{\varrho_{1} \varrho_{2}} e^{\frac{i}{2}\left(\alpha_{1}+\alpha_{2}\right)} s, \sqrt{\varrho_{1} \varrho_{2}} e^{\frac{i}{2}\left(\alpha_{1}-\alpha_{2}\right)} t\right) \\
=\varrho_{1}^{c_{1}-1} \varrho_{2}^{c_{2}-1} e^{i\left(k_{1} \alpha_{1}+k_{2} \alpha_{2}\right)} \mathscr{K}(\tau ; v ; s, t) .
\end{gathered}
$$

Putting $\varrho=\sqrt{\varrho_{1} \varrho_{2}}, \alpha=\frac{1}{2}\left(\alpha_{1}+\alpha_{2}\right), \beta=\frac{1}{2}\left(\alpha_{1}-\alpha_{2}\right)$ we get

$$
\mathscr{K}\left(\tau ; \varrho_{2} v, \varrho e^{i \alpha} S, \varrho e^{i \beta} t\right)=\varrho^{c_{1}-1} \varrho_{2}^{c_{2}-c_{1}} e^{i(k+\alpha+k-\beta)} \mathscr{K}(\tau ; v ; s, t)
$$

where

$$
k_{ \pm}=k_{1} \pm k_{2} .
$$

Because of the one-valuedness of $\mathscr{K}$ either $k_{ \pm}$are integers or $\mathscr{K} \equiv 0$.

Each $\mathscr{K}$ satisfying (2.17) can be written in the form

$$
\mathscr{K}(\tau ; v ; s, t)=u^{c_{1}-1} v^{c_{2}-1} H(\tau ; s, t)
$$

where $u$ is given by (2.9) (we recall that $u$ and $v$ are positive for $p \in V_{+}$so that $H \in D^{\prime}\left(R_{1}^{+} \times \tilde{C}_{2}\right)$ is well defined). Due to (2.17) $H$ satisfies the following simple homogeneity condition

$$
H\left(\tau ; \varrho e^{i \alpha} s, \varrho e^{i \beta}\right)=e^{i k+\alpha} e^{i k_{-} \beta} H(\tau ; s, t) .
$$

Introducing the variables $r, v, \alpha, \beta$ by

$$
s=r \sqrt{\frac{1-v}{2}} e^{i \alpha}, \quad t=r \sqrt{\frac{1+v}{2}} e^{i \beta} \quad(r>0,-1 \leqq v \leqq 1)
$$

and treating for the moment $H$ as an ordinary function we find

$$
H(\tau ; s, t)=e^{i k+\alpha} e^{i k-\beta} H_{1}(\tau, v) .
$$

In order to give a precise meaning of (2.22) in the case when $H$ is a distribution we have to introduce a new class of generalized functions.

To simplify the problem we neglect for the moment the $\tau$-dependence and consider the space $\mathfrak{X}_{k+k-}$ of distributions $\phi(s, t) \in D^{\prime}\left(\tilde{C}_{2}\right)$, satisfying the homogeneity condition (2.20). Introduce the operator $\mathfrak{R}_{k_{+} k_{-}}$defined on the space of test functions $F \in D\left(\tilde{C}_{2}\right)$ by

$$
\begin{aligned}
\left(\Re_{k_{+} k_{-}} F\right)(v)= & \int_{0}^{\infty} r^{3} d r \int_{0}^{2 \pi} d \alpha \int_{0}^{2 \pi} d \beta e^{i k_{+} \alpha} e^{i k-\beta} \\
& \times F\left(r \sqrt{\frac{1-v}{2}} e^{i \alpha}, r \sqrt{\frac{1+v}{2}} e^{i \beta}\right) .
\end{aligned}
$$


$\Re_{k_{+} k_{-}}$defines a continuous mapping $F(s, t) \rightarrow u_{k_{+} k_{-}}(v)=\left(\Re_{k_{+} k_{-}} F\right)(v)$ of $D\left(\tilde{C}_{2}\right)$ onto the space $X_{k_{+} k_{-}}$of test functions $u_{k_{+} k_{-}}$of the form ${ }^{4}$

$$
u_{k_{+} k_{-}}(v)=\left(\frac{1-v}{2}\right)^{\frac{1}{2} \mid k+1}\left(\frac{1+v}{2}\right)^{\frac{1}{2} \mid k-1} v(v)
$$

where $v(v)$ is an infinitely smooth function on $[-1,1]$. We define a topology in $X_{k_{+} k_{-}}$by the (countable) set of semi-norms

$$
\left\|u_{k_{+} k_{-}}(v)\right\|_{n}=\sup \left|\frac{d^{n} v(v)}{d v^{n}}\right|, \quad n=0,1, \ldots,
$$

where $v(v)$ is defined by (2.24).

It can be proved (by a reasoning similar to the one used in Appendix A in the proof of the isomorphism between $\mathfrak{D}_{\chi}^{i}$ and $d_{-\chi}$ ) that each $\phi(s, t)$ $\in \mathfrak{X}_{k_{+} k_{-}}$may be represented in the form

$$
\phi\left(r \sqrt{\frac{1-v}{2}} e^{i \alpha}, r \sqrt{\frac{1+v}{2}} e^{i \beta}\right)=e^{i k_{+} \alpha} e^{i k_{-} \beta} f_{k_{+} k_{-}}(v)
$$

where $f_{k_{+} k_{-}} \in X_{k_{+} k_{-}}^{\prime}$. The exact meaning of (2.26) is given by the equality

$$
(\phi(s, t), F(s, t))=\left(f_{k_{+} k_{-}}(v),\left(\Re_{k_{+} k_{-}} F\right)(v)\right) .
$$

Let further $\mathscr{D} \equiv \mathscr{D}([-1,1])$ be the space of infinitely differentiable functions on the segment $[-1,1]$ with topology defined by the set of semi-norms in the right hand side of (2.25) (see also Appendix E). It is easily seen that $X_{k_{+} k_{-}}^{\prime}$ is isomorphic to $\mathscr{D}^{\prime}$ (the dual of $\mathscr{D}$ ). Namely, to each $g(v) \in \mathscr{D}^{\prime}$ we make correspond to $f_{k_{+} k_{-}}(v) \in X_{k_{+} k_{-}}^{\prime}$ by the following rule: if $u_{k_{+} k_{-}}(v) \in X_{k_{+} k_{-}}$is represented in the form (2.24), then by definition

$$
\left(f_{k_{+} k_{-}}(v), u_{k_{+} k_{-}}(v)\right)=(g(v), v(v)) \text {. }
$$

The generalized function $f_{k_{+} k_{-}}$defined in this way will be written in the form

$$
f_{k_{+} k_{-}}(v)=\left(\frac{1-v}{2}\right)^{-\frac{|k+|}{2}}\left(\frac{1+v}{2}\right)^{-\frac{|k-|}{2}} g(v) .
$$

${ }^{4}$ Let $f(z)$ be an infinitely differentiable function of $z$ and $\bar{z}$ in the complex plane $C_{1}$. Then, it is easy (using the Taylor decomposition of $f$ around the origin) to prove that for any integer $n$

$$
\int_{0}^{2 \pi} e^{i n \alpha} f\left(\varrho e^{i \alpha}\right) d \alpha=\varrho^{|n|} g\left(\varrho^{2}\right),
$$

where $g$ is an infinitely smooth function on $\bar{R}_{1}^{+}$. Applying (twice) this argument to the integral (2.23) we see that the behaviour of $u_{k_{+} k_{-}}$in the neighbourhood of $v= \pm 1$ is given by $(2.24)$. 
Applying this result to $H(\tau ; s, t)$ defined by (2.19) and (2.20) (after smearing it out with a test function of $\tau$ ) we obtain finally, the following theorem on the general form of the invariant kernel $K$.

Theorem 2.2. An invariant distribution $K(p ; z ; w) \in D^{\prime}\left(V_{+} \times \tilde{C}_{2} \times \tilde{C}_{2}\right)$ satisfying the homogeneity condition (2.2) does not vanish only for integer $k_{ \pm}(2.18)$ and in that case has the form

$$
K(p ; z ; w)=(z \tilde{p} \bar{z})^{c_{1}-1}(w \tilde{p} \bar{w})^{c_{2}-1} e^{i k_{+} \alpha} e^{i k_{-} \beta} f(\tau, v)
$$

where $\alpha, \beta, v$ are defined by (2.21) and (2.8):

$$
\alpha=\arg (z \varepsilon w), \quad \beta=\arg (z \tilde{p} \bar{w}), \quad v=\frac{|z \tilde{p} \bar{w}|^{2}-p^{2}|z \varepsilon w|^{2}}{z \tilde{p} \tilde{z} w \tilde{p} \bar{w}},
$$

and

$f(\tau, v)=\left(\frac{1-v}{2}\right)^{-\frac{1}{2} \mid k+1}\left(\frac{1+v}{2}\right)^{-\frac{1}{2}|k-|} g(\tau, v) \in\left[D\left(R_{1}^{+}\right) \otimes X_{k_{+} k_{-}}\right]^{1}$,

$g(\tau, v) \in \mathscr{D}^{\prime}\left(R_{1}^{+} \times[-1,1]\right)$ (the dual space to $\mathscr{D}\left(R_{1}^{+} \times[-1,1]\right)=D\left(R_{1}^{+}\right)$ $\otimes \mathscr{D}([-1,1]))$.

Remark 1. The points $\zeta=\left(\zeta_{1}, \zeta_{2}\right)$ on the unit sphere $\left|\zeta_{1}\right|^{2}+\left|\zeta_{2}\right|^{2}=1$ may be put into one-to-one correspondence with the $2 \times 2$ matrices

$$
\hat{\zeta}=\left(\begin{array}{cc}
\zeta_{1} & \zeta_{2} \\
-\bar{\zeta}_{2} & \bar{\zeta}_{1}
\end{array}\right) \in S U(2)
$$

(then the invariant volume element on the group $S U(2)$ has the form $\left.\frac{1}{4 \pi^{2}} \delta\left(1-|\zeta|^{2}\right)\left|d \zeta_{1} d \bar{\zeta}_{1} d \zeta_{2} d \bar{\zeta}_{2}\right|\right)$. Let $D[S U(2)]$ be the set of infinitely smooth functions on the unit sphere in $C_{2}$ with the natural topology. The homogeneity condition (2.20) with respect to $\varrho$ leads to the following representation:

$$
K(p ; z ; w)=(z \tilde{p} \bar{z})^{c_{1}-1}(w \tilde{p} \bar{w})^{c_{2}-1} T(\tau ; \hat{\zeta})
$$

where $T(\tau ; \hat{\zeta}) \in D^{\prime}\left(R_{1}^{+} \times S U(2)\right)$ and

$$
\zeta=\left(\frac{w \tilde{p} \bar{z}}{\sqrt{z \tilde{p} \bar{z} w \tilde{p} \bar{w}}}, i \sqrt{\frac{p^{2}}{z \tilde{p} \bar{z} w \tilde{p} \bar{w}}} z \in w\right) .
$$

Eq. (2.33) may be useful for the extension of Theorem 2.2 to other cases (for instance, for space like $p$ and $z \tilde{p} \bar{z} w \tilde{p} \bar{w} \neq 0$ it is sufficient to replace $S U(2)$ by $S U(1,1))$. We shall refer to this remark in Section 4.2. 
Remark. Eq. (2.29) becomes formally identical with Eq. (1.14) of Ref. [1], written for $k_{1} \geqq\left|k_{2}\right|$ :

$$
K(p ; z ; w)=(z \tilde{p} \bar{z})^{c_{1}-k_{1}-1}(w \tilde{p} \bar{w})^{c_{2}-k_{1}-1}(z \varepsilon w)^{k_{+}}(z \tilde{p} \bar{w})^{k-} h\left(p^{2} ; v\right),
$$

if we put

$$
h(\tau, v)=\left(\frac{1-v}{2 V \tau}\right)^{-k_{+}}\left(\frac{1+v}{2}\right)^{-k_{-}} g(\tau ; v)
$$

and $v=\cos \theta=(\boldsymbol{n}(z, p), \boldsymbol{n}(w, p))$ where $\boldsymbol{n}(\zeta, p)(\zeta=z$ or $w)$ is the three dimensional unit vector

$$
n_{j}(\zeta, p)=\frac{1}{\zeta \tilde{p} \bar{\zeta}}\left(\sqrt{\tau} \zeta \sigma_{j} \bar{\zeta}-\frac{\zeta \tilde{p} \bar{\zeta}+\sqrt{\tau} \zeta \bar{\zeta}}{\sqrt{\tau}+p^{0}} p_{j}\right) .
$$

\section{The Two-Point Function for Finite-Component Fields}

\subsection{The Invariant Distribution $F(x ; \zeta)$}

The case of finite-dimensional representations is contained in our previous discussion. In fact, if $c-|k|$ is a positive integer the representation $\chi=[k, c]$ is reducible, the set of homogeneous polynomials of $z$ and $\bar{z}$ (of degree $(c+k-1, c-k-1)$ ) forming an invariant $\left(c^{2}-k^{2}\right.$ )dimensional subspace $E_{\chi}$ of the representation space. It is obvious that all results of the preceding section (including representation (2.29)) remain true for this invariant subspace. Our present aim is to study in more detail the two-point function for (irreducible) finite-component fields using the fact that both $F(x ; z ; w)$ and $K(p ; z ; w)$ are polynomials in $z, \bar{z}, w, \bar{w}$. The method of this section makes no use of the spectrum conditions and, hence, applies to the description of the general form of an invariant functional on $D\left(R_{4}\right) \times E_{\chi_{1}} \times E_{\chi_{2}}$. Therefore, we will be able to write down the explicit covariant structure of the two-point function not only in $p$ but also in $x$-space. That will enable us to define the timeordered Green's function in terms of the two-point Wightman functions (Section 3.3).

It is convenient to denote the finite dimensional representations of $S L(2, C)$ by the pair of non-negative (half) integers $(i, j)$ connected with the pair $[k, c]$ by

$$
2 i=c+k-1, \quad 2 j=c-k-1 .
$$

It is well known that the direct product of two irreducible finite-dimensional representations $\left(i_{1}, j_{1}\right)$ and $\left(i_{2}, j_{2}\right)$ can be decomposed into a direct 
sum of irreducible representations:

$$
\left(i_{1}, j_{1}\right) \times\left(i_{2}, j_{2}\right)=\bigoplus_{i=\left|i_{1}-i_{2}\right|}^{i_{1}+i_{2}} \bigoplus_{j=\left|j_{1}-j_{2}\right|}^{j_{1}+j_{2}}(i, j) .
$$

Hence, the study of the two point function $F(x ; z, w)$ of two finitecomponent fields can be reduced to the investigation of a distribution $F(x ; \zeta) \equiv F_{(i, j)}(x ; \zeta)$ with the following properties:

a) As a "function" of $x$ (for fixed $\zeta) F(x ; \zeta) \in D^{\prime}\left(R_{4}\right)$,

b) $F(x ; \zeta)$ is a homogeneous polynomial of $\zeta, \bar{\zeta}\left(\zeta \in C_{2}\right)$ of degree $(2 i, 2 j)$,

c) $F\left(A \tilde{x} A^{*} ; \zeta\right)=F(\tilde{x} ; \zeta A), A \in S L(2, C)$.

Following the procedure of Section 2.1 we derive from (3.3) six differential equations for $F$ :

$$
\begin{gathered}
\left(x^{a \dot{c}} \frac{\partial}{\partial x^{b \dot{c}}}-\zeta_{b} \frac{\partial}{\partial \zeta_{a}}\right) F(x ; \zeta)=0, \quad a=1,2, \quad b=3-a, \\
\left(x^{c \dot{a}} \frac{\partial}{\partial x^{c \dot{b}}}-\bar{\zeta}_{\dot{b}} \frac{\partial}{\partial \bar{\zeta}_{\dot{a}}}\right) F(x ; \zeta)=0, \quad \dot{a}=1,2, \quad \dot{b}=3-\dot{a}, \\
\left(\tau_{3}\right)_{b}^{a}\left(x^{b \dot{c}} \frac{\partial}{\partial x^{a \dot{c}}}-\zeta_{a} \frac{\partial}{\partial \zeta_{b}}\right) F(x ; \zeta)=0, \\
\left(\tau_{3}^{*}\right)_{\dot{b}}^{\dot{a}}\left(x^{c \dot{b}} \frac{\partial}{\partial x^{c \dot{a}}}-\bar{\zeta}_{\dot{a}} \frac{\partial}{\partial \bar{\zeta}_{\dot{b}}}\right) F(x ; \zeta)=0 .
\end{gathered}
$$

Summation has to be carried out over all indices in Eq. (3.5); $\tau_{3}=\tau_{3}^{*}$ $=\left(\begin{array}{rr}1 & 0 \\ 0 & -1\end{array}\right)^{5}$.)

The covariant structure of $F(x ; \zeta)$ can be described in the following way.

Theorem 3.1. Any invariant distribution $F(x ; \zeta)$ satisfying conditions a)-c) above can be written in the form

$$
\begin{aligned}
F_{(i, j)}(x ; \zeta) & =\delta_{i j}(\zeta \tilde{x} \bar{\zeta})^{2 j} F(x) \\
& =\delta_{i j}\left(\zeta \sigma_{\mu} i \frac{\partial}{\partial x_{\mu}} \bar{\zeta}\right)^{2 j} T(x)
\end{aligned}
$$

where $F(x)$ (resp. $T(x)$ ) is a scalar Lorentz invariant distribution of $D^{\prime}\left(R_{4}\right)$. In the non-trivial case $i=j$ for a given $F(x ; \zeta)$ the distribution $F(x)($ resp. $T(x))$ is determined within the accuracy of $2 j$ arbitrary constants.

${ }^{5}$ We use different notations $\left(\sigma_{j}\right)^{a} \dot{b},\left(\tau_{j}\right)_{b}^{a},\left(\tau_{j}^{*}\right)_{\dot{a}}^{\dot{b}}$ for the same numerical matrices because they refer to different transformation properties under $S L(2, C)$. Their equality is basis dependent (it is only invariant under transformations of the $S U(2)$ subgroup of $S L(2, C)$ ). 
More precisely, if $F_{0}(x)$ is a given solution of (3.6), then the general solution has the form

$$
F(x)=F_{0}(x)+\sum_{l=0}^{2 j-1} c_{l}\left(\square_{x}\right)^{l} \delta(x) .
$$

We give the proof of this theorem in Appendix C.

\subsection{Covariant Structure of the Two-Point Function for Finite Dimensional Representations' of $\operatorname{SL}(2, C)$}

Let the covariant two-point function $F(x ; z, w)$ be a homogeneous polynomial in $z, \bar{z}$ and in $w, \bar{w}$ of degree $\left(2 i_{1}, 2 j_{1}\right)$ and $\left(2 i_{2}, 2 j_{2}\right)$, respectively. Using the decomposition (3.2) and Theorem 3.1 we can write

$$
F(x ; z, w)=\sum_{n} \mathscr{P}_{n}^{\left(i_{1} j_{1}, i_{2} j_{2}\right)}(x ; z, w) F_{n}(x),
$$

where $\mathscr{P}_{n}$ is an invariant homogeneous polynomial of degree $n$ in $x$ and of the same degree as $F$ with respect to the remaining variables (it corresponds to the term $(i, j)$ in the right hand side of (3.2) with $i=j=n / 2)$, $F_{n}(x)$ are Lorentz invariant distributions. The summation in (3.9) is carried out in the range $n_{0}=2 \max \left(\left|i_{i}-i_{2}\right|,\left|j_{1}-j_{2}\right|\right) \leqq n=n_{0}+2 k$ $\leqq 2 \min \left(i_{1}+i_{2}, j_{1}+j_{2}\right)$. (If this range is empty then $F$ vanishes identically.) Here $k=0,1, \ldots, k_{\max }$ so that $n$ takes either only even or only odd values. (We mention that a non trivial invariant function $F$ exists only if the difference between $I=i_{1}-i_{2}$ and $J=j_{1}-k_{2}$ is an integer.)

By standard methods of reduction of the direct product of two finitedimensional representations (e.g. using Young's symmetrization and antisymmetrization) we obtain the explicit expression for the polynomial $\mathscr{P}_{n}$ :

$$
\begin{aligned}
& \mathscr{P}_{n}^{\left(i_{1} j_{1}, i_{2} j_{2}\right)}(x ; z, w)=(z \varepsilon w)^{i_{1}+i_{2}-\frac{n}{2}}(\overline{z \varepsilon w})^{j_{1}+j_{2}-\frac{n}{2}} \\
& \cdot\left(z \frac{\partial}{\partial \zeta}\right)^{\frac{n}{2}+i_{1}-i_{2}}\left(w \frac{\partial}{\partial \zeta}\right)^{\frac{n}{2}-i_{1}+i_{2}}\left(\bar{z} \frac{\partial}{\partial \bar{\zeta}}\right)^{\frac{n}{2}+j_{1}-j_{2}}\left(\bar{w} \frac{\partial}{\partial \bar{\zeta}}\right)^{\frac{n}{2}-j_{1}+j_{2}}(\zeta \tilde{x} \bar{\zeta})^{n} \\
& =(z \varepsilon w)^{i_{1}+i_{2}-\frac{n}{2}}(z \varepsilon w)^{j_{1}+j_{2}-\frac{n}{2}}\left(\frac{n}{2}+i_{1}-i_{2}\right) !\left(\frac{n}{2}-i_{1}+i_{2}\right) ! \\
& \cdot\left(\frac{n}{2}+j_{1}-j_{2}\right) !\left(\frac{n}{2}-j_{1}+j_{2}\right) ! \\
& \cdot n ! \sum \frac{(z \tilde{x} \bar{z})^{p_{1}}(z \tilde{x} \bar{w})^{q_{1}}(w \tilde{x} \bar{z})^{p_{2}}(w \tilde{x} \bar{w})^{q_{2}}}{p_{1} ! \quad q_{1} ! \quad p_{2} ! \quad q_{2} !}
\end{aligned}
$$


the summation in (3.10) is carried over those non-negative integers $p_{1}, p_{2}, q_{1}, q_{2}$ for which

$$
\begin{array}{ll}
p_{1}+q_{1}=\frac{n}{2}+i_{1}-i_{2}, & p_{2}+q_{2}=\frac{n}{2}-i_{1}+i_{2}, \\
p_{1}+p_{2}=\frac{n}{2}+j_{1}-j_{2}, & q_{1}+q_{2}=\frac{n}{2}-j_{1}+j_{2} .
\end{array}
$$

We assume that $I=i_{1}-i_{2} \geqq|J|$ where $J=j_{1}-j_{2}$. (The other possibilities are treated analogously.) Putting further $q_{2}=q\left(q_{1}=\frac{n}{2}-J-q\right.$, $\left.p_{1}=J+I+q, p_{2}=\frac{n}{2}-I-q\right)$, dividing and multiplying by $\left(x^{2}|z \varepsilon w|^{2}\right)^{\frac{n}{2}-I}$ and using (3.10) we obtain

$$
\begin{aligned}
& {\left[n !\left(\frac{n}{2}+I\right) !\left(\frac{n}{2}-I\right) !\right]^{-1} \mathscr{P}_{n}^{\left(i_{1} j_{1}, i_{2} j_{2}\right)}(x ; z, w)} \\
& \quad=\left(x^{2}\right)^{\frac{n}{2}-I}(z \varepsilon w)^{2 i_{2}}(\bar{z} \varepsilon \bar{w})^{j_{1}+j_{2}-I}(z \tilde{x} \bar{z})^{I+J}(z \tilde{x} \bar{w})^{I-J} \times P_{\frac{n}{2}-I}^{(I-J, I+J)}(\kappa),
\end{aligned}
$$

where

$$
\kappa=\frac{z \tilde{x} \bar{z} w \tilde{x} \bar{w}+|z \tilde{x} \bar{w}|^{2}}{x^{2}|z \varepsilon w|^{2}}=\frac{z \tilde{x} \bar{z} w \tilde{x} \bar{w}+|z \tilde{x} \bar{w}|^{2}}{z \tilde{x} \bar{z} w \tilde{x} \bar{w}-|z \tilde{x} \bar{w}|^{2}}
$$

and $P_{l}^{(\alpha, \beta)}$ is the Jacobi polynomial:

$$
P_{l}^{(\alpha, \beta)}(\kappa)=\sum_{q=0}^{l}\left(\begin{array}{c}
l+\alpha \\
q
\end{array}\right)\left(\begin{array}{c}
l+\beta \\
l-q
\end{array}\right)\left(\frac{\kappa-1}{2}\right)^{l-q}\left(\frac{\kappa+1}{2}\right)^{q} .
$$

A formula analogous to (3.9) and (3.10) can be derived just as well with $\partial / \partial x$ instead of $x$.

\subsection{Application to the Definition of the Time-Ordered Green's Function for Arbitrary Spinor Fields}

Let $\varphi$ and $\psi$ be local finite-component fields transforming under the representations $\left(i_{1}, j_{1}\right)$ and $\left(i_{2}, j_{2}\right)$ respectively, with integer $I-J\left(=i_{1}-i_{2}\right.$ $-j_{1}+j_{2}$ ). Our task will be to show the existence of a Lorentz invariant time-ordered Green's function $G_{\varphi \psi}(x ; z, w)$ defined by

$$
\left.\begin{array}{l}
G_{\varphi \psi}(x ; z, w)=F_{\varphi \psi}(x ; z, w) \equiv\left\langle 0\left|\varphi\left(\frac{x}{2} ; z\right) \psi\left(-\frac{x}{2} ; w\right)\right| 0\right\rangle \\
\text { for } x^{0}>0,
\end{array}\right\}
$$


where

$$
\sigma=(-1)^{2 k_{1}}\left(=(-1)^{2 k_{2}}\right), \quad k_{\alpha}=i_{\alpha}-j_{\alpha}, \quad \alpha=1,2 .
$$

Locality implies that

$$
F_{\varphi \psi}(x ; z, w)=\sigma F_{\psi \varphi}(-x ; w, z) \text { for } x^{2}<0 .
$$

We shall again make use of the reduction (3.2) and (3.9), so first we study the reduced problem.

Let $F_{1}(x ; \zeta)$ and $F_{2}(x ; \zeta)$ be two distributions satisfying conditions a) - c) of Section 3.1 with $i=j=n / 2$ and let further the locality-type condition

$$
F_{1}(x ; \zeta)=F_{2}(x ; \zeta) \text { for } x^{2}<0
$$

be fulfilled. We shall prove that there exists an invariant distribution $F(x ; \zeta)$, which satisfies a)-c), such that

$$
\begin{array}{ll}
F(x ; \zeta)=F_{1}(x ; \zeta) \quad \text { for } \quad x \in \mathscr{V}_{+} \equiv R_{4} \backslash \bar{V}_{-}, \\
F(x ; \zeta)=F_{2}(x ; \zeta) \text { for } \quad x \in \mathscr{V}_{-} \equiv-\mathscr{V}_{+} .
\end{array}
$$

To do this we use representation (3.6) for $F_{1,2}(x ; \zeta)$ :

$$
F_{\alpha}(x ; \zeta)=(\zeta \tilde{x} \bar{\zeta})^{n} F_{\alpha}(x), \quad \alpha=1,2
$$

where $F_{\alpha}(x)$ are Lorentz invariant distributions and

$$
F_{1}(x)=F_{2}(x) \text { for } x^{2}<0 .
$$

Thus, our problem is reduced to the solved [9] problem of existence of a scalar function $F(x)$ satisfying

$$
F(x)=F_{1}(x) \quad \text { for } \quad x \in \mathscr{V}_{+}, \quad F(x)=F_{2}(x) \text { for } x \in \mathscr{V}_{-} .
$$

[Eq. (3.20) defines an invariant distribution in $\tilde{R}_{4}=R_{4} \backslash\{0\}$. In view of Proposition C.1 (Appendix C) it can be continued to a Lorentz invariant distribution in the whole $R_{4}$.]

In the case when $F_{1,2}(x ; \zeta) \in S^{\prime}\left(R_{4}\right)$ and when the strong spectrum condition

$$
\operatorname{supp} \tilde{F}_{1}(p ; \zeta) \subset V_{+}, \quad \operatorname{supp} \tilde{F}_{2}(p ; \zeta) \subset V_{-}
$$

is satisfied it is more convenient to use the representation (3.7) for $F_{1,2}$ and $F(x ; \zeta)$ :

$$
\begin{array}{ll}
F_{\alpha}(x ; \zeta)=\left(\zeta \tilde{\partial}_{x} \bar{\zeta}\right)^{n} T_{\alpha}(x), & \alpha=1,2 \\
F(x ; \zeta)=\left(\zeta i \tilde{\partial}_{x} \bar{\zeta}\right)^{n} T(x) & \left(\tilde{\partial}_{x} \equiv \sigma_{\mu}-\frac{\partial}{\partial x_{\mu}}\right)
\end{array}
$$


The functions $T_{\alpha}$ and $T$ are determined by (3.22) within the accuracy of arbitrary polynomials in $x^{2}$ of degree ${ }^{6} n-1$. They can be chosen in such a way that

$$
T(x)=T_{1}(x) \text { for } x \in \mathscr{V}_{+}, \quad T(x)=T_{2}(x) \text { for } x \in \mathscr{V}_{-} .
$$

Using the spectrum condition (3.21) we can get rid of the polynomial arbitrariness in the choice of $T_{\alpha}$ and $T$ by requiring fast decrease of these functions for large space-like $x$ :

$$
T_{\alpha}(x)=h_{\alpha}\left(x^{2}\right) \text { for } x^{2}<0 ; \quad h_{\alpha}(\tau) \rightarrow 0 \text { for } \tau \rightarrow-\infty .
$$

(We remind that $h_{\alpha}(\tau)$ is an analytic function for $\tau<0$ so that the limit in (3.24) can be understood in the usual sense.) Indeed, $\tilde{F}_{\alpha}(p ; \zeta)=(\zeta \tilde{p} \bar{\zeta})^{n} \tilde{T}_{\alpha}(p)$ and, hence, in view of (3.21) $\tilde{T}_{\alpha}(p)$ can be chosen in such a way that $\operatorname{supp} \tilde{T}_{1,2}(p) \in V_{ \pm}$. For this choice of $T_{\alpha}$ the boundary condition (3.24) is fulfilled automatically as can be seen from the representation

$$
h_{\alpha}\left(x^{2}\right)=\frac{1}{\sqrt{-x^{2}}} \int_{\mu^{2}}^{\infty} K_{1}\left(\sqrt{-\lambda x^{2}}\right) \varrho_{\alpha}(\lambda) d \lambda \text { for } x^{2}<0,
$$

where $K_{1}(z)$ is the first Macdonald function and $\mu>0$ (see also [10]). One can prove that $T_{\alpha}(x)$ goes to zero for $x^{2} \rightarrow-\infty$ also, for a weaker form of the spectrum condition: $\operatorname{supp} \tilde{F}_{1,2}(p ; \zeta) \subset \bar{V}_{ \pm}$under the additional assumption that $\tilde{F}_{\alpha}(p ; \zeta)$ are measures in $R_{4}$ vanishing for $p=0$ (this last condition is non-trivial only for $n=0$ ).

Now we come to the general existence problem for the invariant Green function satisfying (3.14). Using (3.9) and arguing in the same way as above we obtain

$$
G_{\varphi \psi}(x ; z, w)=\sum_{n} \mathscr{P}_{n}^{\left(i_{1} j_{1}, i_{2} j_{2}\right)}\left(\frac{\partial}{\partial x} ; z, w\right) G_{n}(x)
$$

where $G_{n}(x)$ are Lorentz invariant scalar distributions. Eq. (3.14) determine $G_{\varphi \psi}$ within the accuracy of an invariant distribution with support at the origin. Even if we fix this arbitrariness the distributions $G_{n}(x)$ still are not uniquely defined by (3.26) (each $G_{n}$ being determined only up to a polynomial of $x_{2}$ of degree $\left.n-1\right)$. The choice of $G_{n}$ can be fixed however if we assume as above (in a theory with spectrum conditions) that $G_{n}$ goes to zero for large space-like $x$.

Remark. As well known (see e.g. Streater and Wightman [3], Theorem 4.7) locality (3.15) and spectrum conditions for finite-component fields imply

$$
F_{\varphi \psi}(x ; z, w)=(-1)^{\frac{1}{2} f+2 I} F_{\psi \varphi}(x ; w, z)
$$

${ }^{6}$ See Appendix C (in particular Eq. (C.12)). 
where $f$ is the number of anticommuting (Fermion) fields and $I$ has the same meaning as in (3.11). In our case $(-1)^{2 I+\frac{1}{2} f}=\sigma(-1)^{n_{0}}$ where $n_{0}$ is the lower limit of the summation in (3.9) and $\sigma$ is the sign in (3.15). Indeed, using the fact that the scalar distributions $F_{n}(x)$ in the decomposition (3.9) are even functions of $x$ for $x^{2}<0$, we readily get the following equivalent form of the locality condition (3.15):

$$
F_{\varphi \psi}(x ; z, w)=\sigma(-1)^{n 0} F_{\psi \varphi}(x ; w, z) \text { for } x^{2}<0 .
$$

Because of the spectrum conditions both sides can be continued analytically in the forward tube $R_{4}+i V_{+}$. Going afterwards to the real limit we obtain (3.27) for any $x$. Eq. (3.27) allows us to fix the freedom in the definition of the Green's function (3.14) in the origin in such a way that

$$
G_{\varphi \psi}(x ; z, w)=\sigma(-1)^{n_{0}} G_{\psi \varphi}(x ; w, z) .
$$

Using (3.27) and the properties of the polynomials (3.10) one can derive the following representation for the vacuum expectation value of the fields (anti-)commutator:

$$
\begin{aligned}
\left\langle 0\left|\left[\varphi\left(\frac{x}{2}, z\right), \psi\left(-\frac{x}{2}, w\right)\right]_{-\sigma}\right| 0\right\rangle & \\
& =\sum_{n} \mathscr{P}_{n}^{\left(i_{1} j_{1}, i_{2} j_{2}\right)}\left(\frac{\partial}{\partial x} ; z, w\right) D_{n}(x ; \varphi, \psi)
\end{aligned}
$$

where $D_{n}$ are odd Lorentz invariant scalar distributions. In view of [7] (Section 9) each $D_{n}$ may be written in the form

$$
D_{n}(u ; \varphi, \psi)=\int_{0}^{\infty} D_{\lambda}(u) \varrho_{n}(\lambda ; \varphi, \psi) d \lambda
$$

where $D_{\lambda}(x)$ is the Pauli-Jordan function (i.e. the commutator of a free

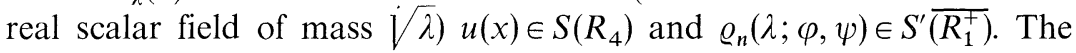
existence of a unitary representation $U(a, 1)$ of the group of translations satisfying (1.1) implies that $\tilde{F}(p ; z, w)$ is a measure on $R_{4}$ (or, equivalently, that $\varrho_{n}(\lambda)$ in (3.30) are measures on $R_{1}$ ). We mention that for $\varphi=\psi$ the representation (3.29) is a consequence of Lorentz invariance alone (locality and spectrum conditions are not needed for its derivation, because in this case (3.27) is a simple consequence of (3.9) and (3.10)).

\section{Decomposition of the Two-Point Function with Respect to Spin}

\subsection{Elementary Invariant Kernels and Poincaré Decomposition of the Two-Point Function}

Now we return to the study of the spectral function (1.3) for arbitrary fields $\varphi$ and $\psi$ transforming according to the irreducible representations $\chi_{1}$ and $\chi_{2}$ (in a theory with a mass gap).

21 Commun math Phys, Vol. 14 
Let $\mathfrak{S}_{\varphi^{*}}$ and $\mathfrak{Y}_{\psi}$ be subspaces in the Hilbert space of states defined as the norm closure of the linear hull of vectors of the type

$$
\varphi(u ; f)^{*}|0\rangle \text { and } \psi(v ; g)|0\rangle \text {, }
$$

respectively, where $u$ and $v$ vary in $S\left(R_{4}\right)$ and $f \in \mathfrak{D}_{-\chi_{1}}, g \in \mathfrak{D}_{-\chi_{2}}$. Let $\mathfrak{H}_{1}$ be the set of their common elements

$$
\mathfrak{H}_{1}=\mathfrak{H}_{\varphi^{*}} \cap \mathfrak{H}_{\psi} .
$$

The unitary representation $U(a, \mathrm{~A})$ of the quantum mechanical Poincaré group (satisfying (1.1)) is, in general, reducible in $\mathfrak{S}_{1}$. The decomposition of $\mathfrak{H}_{1}$ into irreducible invariant subspaces gives rise to a spectral representation of the two-point function which is a generalization of the wellknown representation [11].

Let $\varphi$ and $\psi$ transform under the irreducible representations $\left[k_{1}, c_{1}\right]$ and $\left[k_{2}, c_{2}\right]$ of $S L(2, C)$, respectively $\left(k_{1}-k_{2}\right.$ being an integer). Then it can be shown that the representation $U(a, A)$ is simply reducible in $\mathfrak{S}_{1}$ so that we can write ${ }^{7}$

$$
\mathfrak{S}_{1}=\bigoplus_{s \geqq k} \int^{\oplus} \mathfrak{S}_{m s} d \varrho_{s}(m)
$$

where $k=\max \left(\left|k_{1}\right|,\left|k_{2}\right|\right)$, and $\varrho_{s}(m)(s=k, k+1, \ldots)$ are positive measures on $(0, \infty)$. Each of the irreducible spaces $\mathfrak{H}_{m s}$ can be identified with the space of functions $\Psi_{m s}(p ; \zeta)$ on the hyperboloid $H_{m}^{+}=\left\{p \mid p^{2}=m^{2}, p^{0}>0\right\}$ which are homogeneous polynomials in $\zeta \in C_{2}$ of degree $2 \mathrm{~s}$. The scalar product in $\mathfrak{S}_{m s}$ is defined by

$$
\left\langle\Phi_{m s} \mid \Psi_{m s}\right\rangle_{m s}=\int_{H_{m}^{+}}\left(\frac{\partial}{\partial \bar{\zeta}} \underset{\sim}{p} \frac{\partial}{\partial \zeta}\right)^{2 s} \overline{\Phi_{m s}(p ; \zeta)} \Psi_{m s}(p ; \zeta) \frac{d^{3} \boldsymbol{p}}{\sqrt{m^{2}+p^{2}}}
$$

where

$$
\underset{p}{p}=\varepsilon\left({ }^{t} \tilde{p}\right) \varepsilon^{-1} .
$$

The decomposition (4.3) makes correspond to each $\Phi \in \mathfrak{H}_{1}$ a vector function (depending on the variables $m$ and $s$ ) $\Phi_{m s} \equiv E_{m s} \Phi \in \mathfrak{S}_{m s}$ such that

$$
\langle\Phi \mid \Psi\rangle=\sum_{s \geqq k} \int\left\langle E_{m s} \Phi \mid E_{m s} \Psi\right\rangle_{m s} d \varrho_{s}(m) .
$$

We shall write, in particular,

$$
\begin{aligned}
E_{m s} \Pi_{1} \varphi(u ; f)^{*}|0\rangle & =\Phi_{m s}(p, \zeta ; u, f) \\
E_{m s} \Pi_{1} \psi(v ; g)|0\rangle & =\Psi_{m s}(p, \zeta ; v, g) .
\end{aligned}
$$

${ }^{7}$ Concerning the definition of the decomposition of a unitary representation of a group in a direct integral see [2] (Section 4.3.7). 
where $\Pi_{1}$ is the projection operator on $\mathfrak{H}_{1}$. Translation invariance implies

$$
\begin{aligned}
& \overline{\Phi_{m s}(p, \zeta ; u, f)}=u(p) \chi_{1}(p ; \bar{\zeta} ; f), \\
& \Psi_{m s}(p, \zeta ; v, g)=v(p) \chi_{2}(p ; \zeta ; g) .
\end{aligned}
$$

The invariant kernels $\chi_{j}(p ; \zeta ; z)$ (corresponding to the functionals $\chi_{j}$ ) are homogeneous distributions of $z \in \tilde{C}_{2}$ of indices $\left[k_{j}, c_{j}\right]$. Taking into account that $\chi_{j}$ is a homogeneous polynomial in $\zeta$ (of degree $2 s$ ) we find that $\chi_{j} \neq 0$ only for $s-\left|k_{j}\right|=0,1, \ldots$, and in that case

$$
\chi_{j}(p ; \zeta ; z)=A_{j}(z \tilde{p} \bar{z})^{c_{j}-s-1}(\zeta \tilde{p} \bar{z})^{s-k_{j}}(\zeta \varepsilon z)^{s+k_{j}} .
$$

(We mention that (4.11) is a special case of (2.29) or (2.35).)

Inserting (4.9)-(4.11) in the integrand of the scalar product (4.4) we obtain the contribution from $\mathfrak{S}_{m s}$ to the invariant kernel $K(p ; z, w)(2.29)$ of the two-point function (1.3):

$$
\begin{aligned}
K_{m s}(p ; z, w)= & A_{m s} \delta_{m}^{+}(p)\left(\frac{\partial}{\partial \bar{\zeta}} p \frac{\partial}{\partial \zeta}\right)^{2 s}(z \tilde{p} \bar{z})^{c_{1}-s-1}(w \tilde{p} \bar{w})^{c_{2}-s-1} \\
& \times(\overline{\zeta \varepsilon z})^{s-k_{1}}(z \tilde{p} \bar{\zeta})^{s+k_{1}}(\zeta \varepsilon w)^{s+k_{2}}(\zeta \tilde{p} \bar{w})^{s-k_{2}},
\end{aligned}
$$

where $\delta_{m}^{+}(p)=\theta\left(p_{0}\right) \delta\left(p^{2}-m^{2}\right)$. Proceeding in the same way as in the derivation of (3.11) we obtain the following expression for the elementary invariant kernel $K_{m s}$ (in the case $\left.k_{1} \geqq\left|k_{2}\right|\right)$ :

$$
\begin{aligned}
& K_{m s}(p ; z, w)=B_{m s} \delta_{m}^{+}(p)(z \tilde{p} \bar{z})^{c_{1}-k_{1}-1}(w \tilde{p} \bar{w})^{c_{2}-k_{1}-1} \\
& \times(z \varepsilon w)^{k_{1}+k_{2}}(z \tilde{p} \bar{w})^{k_{1}-k_{2}} P_{s-k_{1}}^{\left(k_{1}+k_{2}, k_{1}-k_{2}\right)}(v)
\end{aligned}
$$

where $v$ is given by $(2.30), P_{n}^{(\alpha, \beta)}(v)$ are the Jacobi polynomials and $B_{m s}=m^{2\left(s+k_{1}\right)}(2 s) !\left(s+k_{1}\right) !\left(s-k_{1}\right) ! A_{m s}$.

The invariant kernel (2.29) can be expanded in terms of the elementary kernels (4.13):

$$
K(p ; z, w)=\sum_{s \geqq k} \int K_{m s}(p ; z, w) d \varrho_{s}(m) .
$$

This expansion corresponds to the following decomposition of $f(\tau ; v)$ (see (2.29) and (2.37))

where

$$
f(\tau, v)=\sum_{s \geqq k} f_{s}(\tau) P_{k_{1},-k_{2}}^{s}(v)
$$

$$
k=\max \left(\left|k_{1}\right|,\left|k_{2}\right|\right)
$$

$\left\{f_{s}(\tau)\right\}$ is a sequence of complex valued measures and $P_{k l}^{s}(v)$ are the generalized spherical functions [12] related to the Jacobi polynomials by

$P_{k l}^{s}(v)=i^{k-l} \sqrt{\frac{(s-k) !(s+k) !}{(s-l) !(s+l) !}}\left(\frac{1-v}{2}\right)^{\frac{1}{2}(k-l)}\left(\frac{1+v}{2}\right)^{\frac{1}{2}(k+l)} P_{s-k}^{(k-l, k+l)}(v)$. 
[In contradistinction to (4.13) the representation (4.15) is valid for any $k_{1}, k_{2}$ (not only for $k_{1} \geqq\left|k_{2}\right|$ ). As shown in Vilenkin [12], Section 3.3.6, $P_{k_{1},-k_{2}}$ depend only on $\left|k_{+}\right|$and $\left.\left|k_{-}\right| \cdot\right]$

We prove in Appendix E that each distribution $f(v) \in X_{k_{+} k_{-}}^{\prime}$ (see Section 2.3) can be expanded in a series of the type (4.15) with a sequence of coefficients $f_{s}$ of polynomial growth (with respect to $s$ ). In our case $f(\tau ; v) \in\left(D\left(R_{1}^{+}\right) \otimes X_{k_{+} k_{-}}\right)^{\prime}$ depends also on $\tau$ and the expansion (4.15) makes sense if and only if the sequence of distributions $f_{s}(\tau) \in D^{\prime}\left(R_{1}^{+}\right)$is polynomially bounded in $s$ (i.e. if

$$
C_{s}(u)=\left(f_{s}(\tau), u(\tau)\right) \quad s=k, k+1, \ldots
$$

is polynomially bounded numerical sequence (in $s$ ) for each test function $\left.u \in D\left(R_{1}^{+}\right)\right)$.

\subsection{Remark on the Positive-Definiteness Condition}

The positive-definiteness of the metric in the Hilbert space imposes the following condition on the spectral function for two Hermitian conjugate fields $\varphi$ and $\varphi^{*}$ (for which $\left[k_{1}, c_{1}\right]=\left[-k_{2}, \bar{c}_{2}\right] \equiv[k, c]$ ):

$$
\left(K_{\varphi \varphi^{*}}(p ; z, w), F(p ; z) \overline{F(p ; w)}\right) \geqq 0
$$

for any $F \in S\left(R_{4}\right) \otimes D\left(\tilde{C}_{2}\right)$. It turns out that the decomposition (4.14) and (4.15) is a decomposition into elementary positive kernels so that condition (4.18) is equivalent to the positivity of the measures $f_{s}(\tau)$ (for $k_{1}=-k_{2}=k$ ). This is a consequence of our derivation of (4.14). Indeed, the scalar product (4.4) is explicitly positive-definite and hence (4.12) is a positive kernel (for $\left[k_{1}, c_{1}\right]=\left[-k_{2}, \bar{c}_{2}\right]$ ) provided that $A_{m s}>0$ which leads to

$$
f_{s}(\tau) \geqq 0 \text {. }
$$

Another proof of the equivalence between (4.18) and (4.19) can be worked out using the general methods of decomposition of invariant positive Hermitian functionals on $D(\mathfrak{M})$ where $\mathfrak{M}$ is finite dimensional homogeneous space (see [13]). In our case such a positive Hermitian functional is the two-point function in coordinate space

$$
W(x, z \mid y, w)=F_{\varphi \varphi^{*}}(x-y ; z, w) .
$$

The role of the homogeneous space $\mathfrak{M}$ (with respect to the group of Poincaré) plays the direct product $R_{4} \times \tilde{C}_{2}$. We are assuming in addition that $F$ is a tempered distribution with respect to its first argument in order to be able to perform Fourier transformation (without introducing analytic functionals). If we restrict ourselves to the $p$-space only (as we actually did in Sections 2 and 4.1) then the whole treatment can be generalized to non-tempered spectral functions in momentum space. 
Using our Remark 1 at the end of Section 2 we can give a purely group theoretical interpretation of the decomposition (2.33). Indeed, in terms of the distribution $T(\tau ; \hat{\zeta}) \in D^{\prime}\left(R_{1}^{+} \times S U(2)\right)$ defined by (2.33) and (2.34) we can rewrite (4.15) in the form

$$
T(\tau ; \hat{\zeta})=\sum_{s \geqq k} f_{s}(\tau) D_{k k}^{(s)}(\hat{\zeta})
$$

where $D_{m n}^{(s)}(\hat{\zeta})$ are the matrix elements of the representation $(s)$ of $S U(2)$ in the canonical basis (see [12] and Appendix B in [1]). By using standard methods of harmonic analysis on $S U(2)$ and the argument in Appendix $\mathrm{E}$ one can obtain a generalization of Theorem E.1 to the case of arbitrary distributions $T(\hat{\zeta}) \in D^{\prime}[S U(2)]$ (not necessarily of the type (4.21)).

We mention finally that (4.15) implies the following extremal property of the generalized spherical functions $P_{k k}^{s}(v)$ (or of the corresponding Jacobi polynomials $P_{s-k}^{(0,2 k)}(v)$ see (4.17)). Take for simplicity the special case when $K$ in (4.18) is a continuous function of $p$ and fix the vector $p$ along the time axis. The preceding discussion implies the following result. Let $C_{k}^{+}$be the convex cone of generalized functions $f(v) \in X_{0,2 k}^{\prime}$ satisfying the positivity condition

$$
\iint(z \bar{w})^{2 k} f\left(|z \bar{w}|^{2}-|z \varepsilon \bar{w}|^{2}\right) F(z) \overline{F(w)} d^{4} z d^{4} w \geqq 0 .
$$

Then any extreme vector ${ }^{8}$ of this cone is proportional (with a positive factor of proportionality) to one of the generalized spherical function $P_{k k}^{s}(v)(s \geqq|k|)$. In the special case $k=0$, when $P_{00}^{s}(v)=P_{s}^{(0,0)}(v)=P_{0}(v)$ are the Legendre polynomials, this result reduces to a known property of the zonal harmonics (see e.g. [14]).

Acknowledgement. The second named author (I. T. T.) would like to thank Dr. C. Kaysen for his hospitality at the Institute for Advanced Study in Princeton where the present paper was completed.

\section{Appendix A. Isomorphism between $\mathfrak{D}_{x}^{\prime}$ and the Subspace of Homogeneous Distributions of Index $-\chi$ of $D^{\prime}\left(\tilde{C}_{2}\right)$}

\section{A.1. Synopsis on the Decription of the Irreducible Representations} of $S L(2, C)$ in Terms of Homogeneous Functions $([2])$

Let $\mathfrak{D}_{\chi}$ be the space of single-valued infinitely differentiable functions of $z=\left(z_{1}, z_{2}\right) \in \tilde{C}_{2}=C_{2} \backslash\{0\}$ homogeneous of index $\chi=[k, c]$ :

$$
\begin{aligned}
f(\sqrt{\varrho} z) & =\varrho^{c-1} f(z) \text { for } \varrho>0, \\
f\left(e^{i \frac{\alpha}{2}} z\right) & =e^{i k \alpha} f(z) \text { for } \alpha \text { real } .
\end{aligned}
$$

${ }^{8}$ We recall that a point $x$ of a convex set $C$ is called an extreme point of $C$ if it is not an internal point of any segment lying in $C$. A vector $x$ of a convex cone $K$ is called extreme (or non-decomposable) in $K$ if it defines an extreme point in the intersection of $K$ with a hyperplane which does not cross the origin. 
The condition of single-valuedness implies that $k$ is integer or halfinteger; $c$ is in general an arbitrary complex number.

We introduce topology in $\mathfrak{D}_{\chi}$ by the denumerable set of norms $\|f\|_{n}=\sup _{|z|=1} \max _{\Sigma\left(\left|\alpha_{i}\right|+\left|\beta_{i}\right|\right) \leqq n}\left|\left(\frac{\partial}{\partial z_{1}}\right)^{\alpha_{1}}\left(\frac{\partial}{\partial z_{2}}\right)^{\alpha_{2}}\left(\frac{\partial}{\partial \bar{z}_{1}}\right)^{\beta_{1}}\left(\frac{\partial}{\partial \bar{z}_{2}}\right)^{\beta_{2}} f\left(z_{1}, z_{2}\right)\right|$,

where

$$
|z|^{2}=z_{1} \bar{z}_{1}+z_{2} \bar{z}_{2} \text {. }
$$

(With this topology $\mathfrak{D}_{\chi}$ is a complete nuclear space.)

The representation $\chi$ of $S L(2, C)$ is defined in $\mathfrak{D}_{\chi}$ by

$$
\left[T_{\chi}(A) f\right](z)=f\left({ }^{t} A z\right) \equiv f(z A) .
$$

This representation is irreducible in $\mathfrak{D}_{\chi}$ if and only if the pair $[k, c]$ does not satisfy one of the following relations:

$$
\begin{aligned}
c-|k| & =1,2, \ldots, \\
-c-|k| & =1,2, \ldots .
\end{aligned}
$$

In the exceptional cases (A.4) the representation $\chi$ has a generalized triangular form: it is reducible but not fully reducible (i.e. decomposable) [2]. In the case (A.4a) the space $\mathfrak{D}_{\chi}$ contains the finite-dimensional invariant subspace $E_{\chi}$ of homogeneous polynomials of $z$ and $\bar{z}$. For all $\chi$ different from (A.4) the representations $\chi$ and $-\chi$ are equivalent [2].

\section{A.2. The Mapping of $D\left(\tilde{C}_{2}\right)$ on $\mathfrak{D}_{\chi}$ and its Adjoint}

We define the continuous linear mapping $I_{\chi}$ from the Schwartz space of test functions $F \in D\left(\tilde{C}_{2}\right)$ onto $\mathfrak{D}_{\chi}(\ni f)$ :

$$
f(z)=\left(I_{\chi} F\right)(z)=\frac{1}{2} \int_{0}^{\infty} d \varrho \int_{0}^{4 \pi} d \alpha F\left(\sqrt{\varrho} e^{\frac{2}{2} \alpha} z\right) \varrho^{-c} e^{-i k \alpha} .
$$

To prove that the image of $D\left(\tilde{C}_{2}\right)$ under the mapping (A.5) is the whole space $\mathfrak{D}_{\chi}$ we choose some $h(\varrho) \in D(0, \infty)$ for which

$$
2 \pi \int_{0}^{\infty} \frac{h(\varrho)}{\varrho} d \varrho=1
$$

and for any given $f \in \mathfrak{D}_{\chi}$ put

$$
F(z)=f(z) h\left(|z|^{2}\right) .
$$

With such $F$ Eq. (A.5) is verified immediately. (It is clear that the mapping $I_{\chi}$ is not one-to-one.)

Now we define the adjoint mapping $\varphi \rightarrow \Phi=I_{\chi}^{*} \varphi$ from $\mathfrak{D}_{\chi}^{\prime}$ into $D^{\prime}\left(\tilde{C}_{2}\right)$ by

$$
(\Phi, F)=\left(\varphi, I_{\chi} F\right)\left(F \in D\left(\tilde{C}_{2}\right)\right) .
$$


It is easily checked that the $\Phi$ defined by (A.8) belongs to the space $d_{-\chi}$ of homogeneous distributions of index of homogeneity $-\chi=[-k,-c]$ : for $a=\sqrt{\varrho} e^{i \frac{\alpha}{2}}$

Indeed,

$$
\Phi(a z)=\varrho^{-c-1} e^{-i k \alpha} \Phi(z) .
$$

$$
\begin{aligned}
(\Phi(a z), F(z)) & =\frac{1}{\varrho^{2}}\left(\Phi(z), F\left(\frac{z}{a}\right)\right)=\frac{1}{\varrho^{2}}\left(\varphi, I_{\chi} F\left(\frac{z}{a}\right)\right) \\
& =\frac{e^{-i k \alpha}}{\varrho^{c+1}}\left(\varphi, I_{\chi} F(z)\right)=\varrho^{-c-1} e^{-i k \alpha}(\Phi, F) .
\end{aligned}
$$

We shall prove that in fact the mapping $I_{\chi}^{*}$ is an isomorphism between $\mathfrak{D}_{\chi}^{\prime}$ and $d_{-\chi}$. We have seen already that for each $\varphi \in \mathfrak{D}_{\chi}^{\prime}$ there is $a \Phi=I_{\chi}^{*} \varphi \in d_{-\chi}$. Now we shall need the following lemma.

Lemma A.1. Each distribution $\Phi \in D^{\prime}\left(\tilde{D}_{2}\right)$ satisfying the homogeneity condition (A.9) vanishes on the subspace

$$
\mathscr{E}_{\chi}=\left\{F(z) \in D\left(\tilde{C}_{2}\right) \mid I_{\chi} F(z) \equiv 0\right\}
$$

(i.e. on the kernel of the mapping (A.5)).

Proof $^{9}$. In view of (A.9) for any two test functions $F(z), G(z) \in D\left(\tilde{C}_{2}\right)$ the following identity holds:

$$
\begin{aligned}
\varrho^{-1} \cdot\left(\Phi(z), F(z) \cdot G\left(\frac{z}{a}\right)\right)[\equiv \varrho(\Phi(a z), F(a z) G(z))] \\
=\varrho^{-c} e^{-i k \alpha}(\Phi(z), F(a z) G(z)), \quad\left(a=\sqrt{\varrho} e^{i \frac{\alpha}{2}}\right) .
\end{aligned}
$$

We integrate both sides of (A.12) with $|d a d \bar{a}|=\frac{1}{2} d \varrho d \alpha$. Then the lefthand side can be interpreted as the value of the distribution $H(z ; a)$ $=\Phi(z) \times \frac{1}{\varrho} \in D^{\prime}\left(\tilde{C}_{2} \times \tilde{C}_{1}\right)$ applied to the test function $F(z) G\left(\frac{z}{a}\right) ;$ an analogous meaning can be given also to the right-hand side. In view of the commutativity of the direct product of distributions we obtain

$$
\left(\Phi(z), F(z) \int G\left(\frac{z}{a}\right) \frac{d \varrho}{2 \varrho} d \alpha\right)=\left(\Phi(z), G(z)\left(I_{\chi} F\right)(z)\right)
$$

9 The argument goes similarly to Araki's proof of Lemma 2 of [15] (p. 272). This lemma of Araki may appear useful for the study of Lorentz invariant distributions of $n$ 4-vectors. Therefore it would be of interest to refine its original proof: Araki assumed that for any $f(x) \in D\left(R_{4 n}\right)$ the function $\breve{f}(x)=\int f(\Lambda x) s \Lambda$ obtained from $f$ by integrating over the Lorentz group is a multiplicator in $D\left(R_{4 n}\right)$. One can only clame that $\breve{f}(x)$ is a multiplicator in $D\left(R_{4 n} \backslash\{0\}\right)$; in general $\breve{f}(x)$ may be singular at $x=0$. Hence, the possibility of going to the limit $R \rightarrow \infty$ in the identity

$$
\left(F, \int_{\|\Lambda\|<R} f(\Lambda x) g(x) d \Lambda\right)=\left(F, \int_{\|\Lambda\|<R} f(x) g\left(\Lambda^{-1} x\right) d \Lambda\right)
$$

should be studied more carefully. 
where $I_{\chi} F$ is given by (A.5). Now, putting $G(z)=h\left(|z|^{2}\right)$ where $h(\varrho)$ satisfies (A.6), we obtain $(\Phi, F)=0$ if $I_{\chi} F=0$ which proves our statement.

It follows from this lemma that for each $\Phi \in d_{-\chi}$ there exists a linear functional $\varphi$ on $\mathfrak{D}_{\chi}$ such that

$$
(\varphi, f)=(\Phi, F) \text { for } f=I_{\chi} F
$$

(Lemma A.1 shows that the definition (A.14) for $(\varphi, f)$ does not depend on the choice of the solution $F$ of the equation $f=I_{\chi} F$; the functional $\varphi$ is defined on the whole $\mathfrak{D}_{\chi}$ since the image of $D\left(\tilde{C}_{2}\right)$ under $I_{\chi}$ coincides with $\mathfrak{D}_{\chi}$.) The continuity of $\varphi$ is a consequence of some general theorems. It is evident also from the formula:

$$
(\varphi, f)=\left(\Phi(z), h\left(|z|^{2}\right) f(z)\right) .
$$

where $h(\varrho)$ is chosen as in (A.6). Hence, $\varphi \in \mathfrak{D}_{\chi}^{\prime}$.

The representation (A.3) of $S L(2, C)$ in $\mathfrak{D}_{\chi}$ generates a representation $T^{x}(A)$ in $\mathfrak{D}_{\chi}^{\prime}$ by

$$
\left(T_{\chi}^{\prime}(A) \varphi, f\right)=\left(\varphi, T_{\chi}\left(A^{-1}\right) f\right) .
$$

The isomorphism $I_{\chi}^{*}$ between $\mathfrak{D}_{\chi}^{\prime}$ and $d_{-\chi}$ permits us to define the representation of $S L(2, C)$ also in $d_{-\chi}$ by putting

$$
\Phi \rightarrow \Phi_{A}=I_{\chi}^{*} T_{\chi}^{\prime}(A) \varphi, \quad \varphi=\left(I_{\chi}^{*}\right)^{-1} \Phi .
$$

Using (A.8) and (A.16) we obtain

$$
\begin{aligned}
\left(\Phi_{A}(z), F(z)\right) & =\left(T_{\chi}^{\prime}(A) \varphi, I_{\chi} F\right)=\left(\varphi, T_{\chi}\left(A^{-1}\right) I_{\chi} F\right) \\
& =\left(\varphi, I_{\chi}\left[F\left(z A^{-1}\right)\right]\right)=\left(\Phi(z), F\left(z A^{-1}\right)\right)=(\Phi(z A), F(z)),
\end{aligned}
$$

i.e. $\Phi_{A}(z)=\Phi(z A)$.

Thus, the representation (A.17) of $S L(2, C)$ in $d_{-\chi}$ is an extension of the representation $T_{-\chi}$ defined by (A.3) in $\mathfrak{D}_{-\chi}$.

\section{Appendix B. Regularity of Mapping $O_{1} \rightarrow \Omega_{1}$ (Proof of Lemma 2.1)}

It is rather complicated to check directly that the change of variables (2.10) (2.8) is a regular one. In order to simplify the proof we shall perform it in several steps. We shall restrict ourselves to the discussion of the first case (i.e. of the mapping of $O_{1}=V_{+} \times \tilde{C}_{2} \times C_{1} \times \tilde{C}_{1}\left(w_{2} \neq 0\right)$ on $\left.\Omega_{1}\right)$.

Our first substitution is

$$
(\tilde{p} ; z ; w) \rightarrow\left(\tilde{p} ; \zeta=z_{1}+\frac{p^{21}}{p^{11}} z_{2}, \quad z_{2} ; \eta=w_{1}+\frac{p^{21}}{p^{11}} w_{2}, w_{2}\right) .
$$

It is one-to-one, infinitely differentiable mapping of $O_{1}$ onto itself with Jacobian equal to 1 . 
Next we put

$$
\left(\tilde{p} ; \zeta, z_{2} ; \eta, w_{2}\right) \rightarrow\left(\tilde{p} ; s=\sqrt{\tau}\left(\zeta w_{2}-\eta z_{2}\right), \quad t=p^{11} \zeta \bar{\eta}+\frac{\tau}{p^{11}} z_{2} \bar{w}_{2} ; \eta, w_{2}\right)
$$

where $\tau=\operatorname{det} \tilde{p}=p^{2}$. The Jacobian for this (one-to-one, $C^{\infty}$ ) change of variables is

$$
\frac{D(s, \bar{s} ; t, \bar{t})}{D\left(\zeta, \bar{\zeta} ; z_{2}, \bar{z}_{2}\right)}=\left|\frac{D(s, t)}{D\left(\zeta, z_{2}\right)}\right|^{2}=\tau\left(p^{11} \eta \bar{\eta}+\frac{\tau}{p^{11}} w_{2} \bar{w}_{2}\right)^{2}=p^{2}(w \tilde{p} \bar{w})^{2}>0 .
$$

Further, using that $w_{2} \neq 0$ in $O_{1}$ we put

$$
w_{2}=\varrho e^{i \varphi} \quad(\varrho>0,-\infty<\varphi<\infty) .
$$

Thus we establish an isomorphism between $D^{\prime}\left(O_{1}\right)(\ni K(\tilde{p} ; z ; w))$ and the space $D^{\prime}\left(V_{+} \times \tilde{C}_{2} \times C_{1} \times R_{1}^{+} \times R_{1}\right)$ of distributions $\tilde{K}(\tilde{p} ; s, t ; \eta ; \varrho, \varphi)$ periodic of period $2 \pi$ with respect to $\varphi$ (cf. [16]).

Finally, we make one more (regular) change of variables

$$
\left(p^{11}, p^{22}, p^{21} ; s, t ; \eta ; \varrho, \varphi\right) \rightarrow\left(\tau ; p^{11}, p^{21} ; v ; s, t ; \eta ; \varphi\right)
$$

where $v=p^{11} \eta \bar{\eta}+\frac{\tau}{p^{11}} \varrho^{2}(=w \tilde{p} \bar{w})$. This way we come to the set $D^{\prime}\left(\Omega_{1}\right)$ and this completes the proof of Lemma 2.1.

\section{Appendix C. General Form of Covariant Tensor-Valued Distributions (Proof of Theorem 3.1)}

The proof of Theorem 3.1 is based on the following two lemmas.

Lemma C.1. Let $F(x ; \zeta)$ satisfy the conditions a)-c) of Section 3.1. Then there exists a (unique for the non-trivial case $i=j$ ) pair of invariant distributions $f_{ \pm}(\tau) \in D^{\prime}\left(R_{1}\right)$ which coincide for $\tau<0$ and are related to $F(x ; \zeta)$ by

$$
F(x ; \zeta)=(\zeta \tilde{x} \bar{\zeta})^{2 j} f_{ \pm}\left(x^{2}\right) \delta_{i j} \quad \text { for } \quad x \in \mathscr{V}_{ \pm}
$$

where the domains $\mathscr{V}_{ \pm}$are determined in (3.17).

Proof. We shall limit ourselves to the consideration of $F(x ; \zeta)$ in $\mathscr{V}_{+}$. Because of the invariance condition (3.3) it is sufficient to prove (C.1) in the domain

$$
X_{+}=\left\{x \in R_{4} \mid x^{\mu}>0 \mu=0,1,2,3\right\},
$$

since

$$
\mathscr{V}_{+}=\bigcup_{\Lambda \in L_{+}^{\uparrow}} \Lambda X_{+} .
$$


$F(x ; \zeta)$ being a polynomial in $\zeta$ and $\bar{\zeta}$ is uniquely determined as a function of $\zeta$ by its values in the domain

$$
Z_{+}=\left\{\zeta \in C_{2} \mid \zeta \sigma_{\mu} \bar{\zeta}>0, \mu=0,1,2,3\right\} .
$$

In analogy with the reasoning in Section 2.2 we substitute (for fixed $\left.\zeta \in Z_{+}\right)$the variables $x^{11}$ and $x^{22}$ by $\tau=\operatorname{det} \tilde{x}$ and $u=\zeta \tilde{x} \bar{\zeta}$. This change of variables is legitimate in the domain $\left\{x \in X_{+}, \zeta \in Z_{+}\right\}$because it is oneto-one infinitely differentiable, its Jacobian being

$$
\frac{D(u, \tau)}{D\left(x^{11}, x^{22}\right)}=x^{0} \zeta \sigma_{3} \bar{\zeta}+x^{3} \zeta \sigma_{0} \bar{\zeta}>0 .
$$

Substituting $F(x ; \zeta)=G\left(\tau, u ; x^{12} ; \zeta\right)$ in the differential equations (3.4), (3.5) we find

$$
\begin{gathered}
x^{22} \frac{\partial G}{\partial x^{12}}-\zeta_{1} \frac{\partial G}{\partial \zeta_{2}}=0, \quad x^{22} \frac{\partial G}{\partial x^{21}}-\bar{\zeta}_{1} \frac{\partial G}{\partial \bar{\zeta}_{2}}=0, \\
x^{11} \frac{\partial G}{\partial x^{21}}-\zeta_{2} \frac{\partial G}{\partial \zeta_{1}}=0, \quad x^{11} \frac{\partial G}{\partial x^{12}}-\bar{\zeta}_{2} \frac{\partial G}{\partial \bar{\zeta}_{1}}=0, \\
x^{12} \frac{\partial G}{\partial x^{12}}-x^{21} \frac{\partial G}{\partial x^{21}}-\zeta_{1} \frac{\partial G}{\partial \zeta_{1}}+\zeta_{2} \frac{\partial G}{\partial \zeta_{2}}=0, \\
x^{21} \frac{\partial G}{\partial x^{21}}-x^{12} \frac{\partial G}{\partial x^{12}}-\bar{\zeta}_{1} \frac{\partial G}{\partial \bar{\zeta}_{1}}+\bar{\zeta}_{2} \frac{\partial G}{\partial \bar{\zeta}_{2}}=0 .
\end{gathered}
$$

We consider (C.5) as a linear system of six homogeneous equations with respect to the six unknown variables $\frac{\partial G}{\partial x^{12}}, \frac{\partial G}{\partial x^{21}}, \frac{\partial G}{\partial \zeta_{1}}, \frac{\partial G}{\partial \zeta_{2}}$, $\frac{\partial G}{\partial \bar{\zeta}_{1}}, \frac{\partial G}{\partial \bar{\zeta}_{2}}$. Its determinant

$$
\left(x^{22} \zeta_{2} \bar{\zeta}_{2}-x^{11} \zeta_{1} \zeta_{1}\right) \zeta \tilde{x} \bar{\zeta}=-\left(x^{0} \zeta \sigma_{3} \bar{\zeta}+x^{3} \zeta \sigma_{0} \bar{\zeta}\right) \zeta \tilde{x} \bar{\zeta}
$$

is negative in the domain (C.2), (C.4). Hence the derivatives of $G$ with respect to $x^{11}, x^{21}, \zeta$ and $\bar{\zeta}$ vanish in this domain. Therefore in a sufficiently small neighbourhood of any point $\left(x^{(0)}, \zeta^{(0)}\right) \in X_{+} \times Z_{+}$there exists a distribution $g^{(0)}(\tau, u)$ such that $G\left(\tau, u ; x^{12} ; \zeta\right)=g^{(0)}(\tau, u)$ or equivalently

$$
F(x ; \zeta)=g^{(0)}\left(x^{2}, \zeta \tilde{x} \bar{\zeta}\right)
$$

On the other hand the group $S L(2, C)$ of transformations $(\tilde{x}, \zeta)$ $\rightarrow\left(A \tilde{x} A^{*}, \zeta A^{-1}\right)$ acts transitively on the set $\left\{(x ; \zeta) \mid x^{2}=\tau, \zeta \tilde{x} \bar{\zeta}=u>0\right\}$ (see Appendix D) and hence (3.3) and (C.6) imply that $g^{(0)}(\tau, u)$ does not depend on the choice of the point $\left(x^{(0)}, \zeta^{(0)}\right)$. Thus there exists a $g(\tau, u)$ 
$\in D^{\prime}\left(R_{1} \times R_{1}^{+}\right)$, such that

$$
F(x, \zeta)=g\left(x^{2}, \zeta \tilde{x} \bar{\zeta}\right)
$$

The homogeneity condition gives $F(x ; \lambda \zeta)=\lambda^{2 i} \bar{\lambda}^{2 j} F(x ; \zeta)$ for any complex $\lambda$. Therefore $g(\tau, u)=0$ for $i \neq j$ and

$$
g(\tau, u)=u^{2 i} f_{+}(\tau) \text { for } i=j .
$$

The validity of Eq. (C.1) in $\mathscr{V}_{+} \cap \mathscr{V}_{-}$shows that $f_{+}(\tau)=f_{-}(\tau)$ for $\tau<0$. This completes the proof of Lemma C.1.

As a consequence we obtain for $x \neq 0$

$$
F(x ; \zeta)=(\zeta \tilde{x} \bar{\zeta})^{2 j} F(x) \delta_{i j}
$$

where $F(x) \in D^{\prime}\left(\tilde{R}_{4}\right) \equiv D^{\prime}\left(R_{4} \backslash\{0\}\right)$ and $F(x)=f_{ \pm}\left(x^{2}\right)$ for $x \in \mathscr{V}_{ \pm}$:

Next we study invariant functions $F(x ; \zeta)$ with support in the origin.

Lemma C.2. If $F(x ; \zeta)$ satisfies the conditions of Theorem 3.1 and $\operatorname{supp} F(x ; \zeta)=\{x=0\}$ then

$$
F(x ; \zeta)=(\zeta \tilde{x} \bar{\zeta})^{2 j} \sum_{l=2 j}^{N} c_{l} \square^{l} \delta(x) \delta_{i j} .
$$

Proof. Since $F$ has a compact support it belongs to $S^{\prime}\left(R_{4}\right)$ and its Fourier transform $\tilde{F}(p ; \zeta)$ is a polynomial both in $p$ and $(\zeta, \bar{\zeta})$. From the invariance condition (or Lemma C.1) we find easily that

Using the identities

$$
\tilde{F}(p ; \zeta)=(\zeta \tilde{p} \bar{\zeta})^{2 i} \sum_{k=0}^{N^{\prime}} a_{k}\left(p^{2}\right)^{k} \delta_{i j}
$$

we find

$$
\left(\zeta \tilde{\partial}_{p} \bar{\zeta}\right)(\zeta \tilde{p} \bar{\zeta})=0, \quad \zeta \tilde{\partial}_{p} \bar{\zeta} p^{2}=2 \zeta \tilde{p} \bar{\zeta}
$$

$$
\begin{gathered}
\left(\zeta \tilde{\partial}_{p} \bar{\zeta}\right)^{n}\left(p^{2}\right)^{k}=0 \text { for } n>k \\
\left(\zeta \tilde{\partial}_{p} \bar{\zeta}\right)^{n}\left(p^{2}\right)^{k}=\left(\begin{array}{l}
k \\
n
\end{array}\right) 2^{n}(\zeta \tilde{p} \bar{\zeta})^{n}\left(p^{2}\right)^{n-k} \text { for } n \leqq k
\end{gathered}
$$

so that

$$
(\zeta \tilde{p} \bar{\zeta})^{n}\left(p^{2}\right)^{k}=\frac{k ! n !}{(n+k) !} 2^{-n} \cdot\left(\zeta \tilde{\partial}_{p} \bar{\zeta}\right)^{n}\left(p^{2}\right)^{k+n} .
$$

Hence for $i=j=n / 2$

$$
\tilde{F}(p ; \zeta)=\left(\zeta \tilde{\partial}_{p} \bar{\zeta}\right)^{n} \sum_{k=n}^{N} C_{k}\left(p^{2}\right)^{k}
$$

which is equivalent to (C.9).

The representation (3.6) is a simple consequence of Lemma C.1 (or Eq. (C.8)) combined with the following proposition C.1 and with Lemma C.2. 
Proposition C.1. (Gårding and Roos, see [7] Section 8, in particular the proof of Theorem 8.1.). Each scalar Lorentz invariant distribution $F(x) \in D^{\prime}\left(R_{4} \backslash\{0\}\right)$ can be continued to a Lorentz invariant distribution in $D^{\prime}\left(R_{4}\right)$.

For $F(x ; \zeta) \in S^{\prime}\left(R_{4}\right)$ representation (3.7) is trivially obtained by applying (3.6) to the Fourier transform of $F(x ; \zeta)$. In the general case (for $\left.F(x ; \zeta) \in D^{\prime}\left(R_{4}\right)\right)$ let $f_{ \pm}(\tau) \in D^{\prime}\left(R_{1}\right)$ be the distributions defined by (C.1). Then there exists another pair of distributions $h_{ \pm}(\tau) \in D^{\prime}\left(R_{1}\right)$ which coincide for $\tau<0$ and satisfy the equation $\left(\zeta \tilde{\partial}_{x} \bar{\zeta}\right)^{n} h_{ \pm}\left(x^{2}\right)=(\zeta \tilde{x} \bar{\zeta})^{n} f_{ \pm}\left(x^{2}\right)$ for $x \neq 0$, or in view of (C.11) $(d / d \tau)^{n} h_{ \pm}(\tau)=2^{-n} f_{ \pm}(\tau)$. This proves (3.7) for $x \neq 0$. The validity of this representation in the whole $R_{4}$ is then a consequence of Proposition C. 1 and Lemma C.2.

The freedom in the choice of $F(x)$ and $T(x)$ in (3.6) and (3.7) is fixed by (C.12) and (C.13).

\section{Appendix D. $S L(2, C)$ Orbits in $\tilde{R}_{4} \times \tilde{C}_{2}$}

We will study in this appendix the structure of the orbits in the set $\left\{(p, z) \mid p \in \tilde{R}_{4}\left(=R_{4} \backslash\{0\}\right), z \in \tilde{C}_{2}\right\}$ generated by the $S L(2, C)$ transformations

$$
(\tilde{p}, z) \rightarrow\left(A \tilde{p} A^{*}, z A^{-1}\right) .
$$

We shall see in particular that if $p^{2} \leqq 0$ some "singular" orbits appear at the surface $z \tilde{p} \bar{z}=0$. These additional orbits do not play any role in the study of the covariant two-point function for finite-component fields which is a polynomial in $z$. Their existence shows however that the strong form of the spectrum condition is an essential assumption for our discussion of the general case (Section 2 and Appendix B). It may serve also as a preliminary step to the study of the general form of an invariant distribution of $D^{\prime}\left(R_{4} \times \tilde{C}_{2}\right)$. [Similarly, the $S L(2, C)$ orbits in $R_{4} \times \tilde{C}_{2} \times \tilde{C}_{2}$ are of interest for the study of invariant distributions of $\mathfrak{D}^{\prime}\left(R_{4} \times \tilde{C}_{2} \times \tilde{C}_{2}\right)$.]

We say that a set of invariant functions $\left\{\varphi_{j}(p, z)\right\} j=1, \ldots, N$ is complete for some values $a_{j}$ of $\varphi_{j}$ if the invariant surface

$$
\mathfrak{M}\left(a_{1}, \ldots, a_{N}\right)=\left\{(p, z) \in \tilde{R}_{4} \times \tilde{C}_{2} \mid \varphi_{j}(p, z)=a_{j}, j=1, \ldots, N\right\}
$$

is a homogeneous space for the group of transformations (D.1), or, in other words, if $S L(2, C)$ acts transitively on $\mathfrak{M}$, i.e. if for any $(\tilde{p}, z) \in \mathfrak{M}$ and for an arbitrary fixed $(\tilde{q}, \zeta) \in \mathfrak{M}$ there exists an $A \in S L(2, C)$ such that

$$
(\tilde{p}, z)=\left(A \tilde{q} A^{*}, \zeta A^{-1}\right) .
$$

Each invariant function defined on a submanifold of $\tilde{R}_{4} \times \tilde{C}_{2}$ is a function of the complete set of invariants on this submanifold. 
In what follows we shall find a complete set of invariants on any invariant surface defined by the two known invariants

$$
\varphi_{1}(\tilde{p}, z)=\operatorname{det} \tilde{p}=p^{2} \quad \text { and } \quad \varphi_{2}(\tilde{p}, z)=z \tilde{p} \bar{z} .
$$

Proposition D.1. The set of invariants (D.4) is complete if and only if $a_{2}=\varphi_{2}(\tilde{p}, z) \neq 0$.

Proof. Let $\zeta^{\prime} \tilde{q} \bar{\zeta}^{\prime}=\zeta \tilde{\mathrm{q}} \bar{\zeta}=a_{2} \neq 0$. Then there exists a transformation $B \in S L(2, C)$ from the stability subgroup of $\tilde{q}$ (i.e. satisfying $B \tilde{q} \mathrm{~B}^{*}=\tilde{q}$ ) such that $\zeta=\zeta^{\prime} B$. This transformation can be constructed explicitly if we take, e.g., the following special values of $\tilde{q}$ and $\zeta$ :

$$
\begin{aligned}
& \tilde{q}^{\mathrm{I}}=\operatorname{sgn} a_{2} \sqrt{a_{1}}\left(\begin{array}{ll}
1 & 0 \\
0 & 1
\end{array}\right) \quad\left(\text { for } q_{1}^{2}=a_{1}>0\right) \quad \zeta=\sqrt{\frac{\left|a_{2}\right|}{a_{1}}}\left(\begin{array}{l}
1 \\
0
\end{array}\right) ; \\
& \tilde{q}_{\mathrm{II}}=\operatorname{sgn} a_{2}\left(\begin{array}{ll}
1 & 0 \\
0 & 0
\end{array}\right) \quad\left(\text { for } q_{\mathrm{II}}^{2}=a_{1}=0\right) \quad \zeta=\sqrt{\left|a_{2}\right|}\left(\begin{array}{l}
1 \\
0
\end{array}\right) ; \\
& \tilde{q}_{\mathrm{III}}=\operatorname{sgn} a_{2} \sqrt{-a_{1}}\left(\begin{array}{rr}
1 & 0 \\
0 & -1
\end{array}\right) \quad\left(\text { for } q_{\mathrm{III}}^{2}=a_{1}<0\right) \quad \zeta=\sqrt{\frac{\left|a_{2}\right|}{-a_{1}}}\left(\begin{array}{l}
1 \\
0
\end{array}\right) \text {. }
\end{aligned}
$$

The stability subgroup is $S U(2)$ for $\tilde{q}_{\mathrm{I}}, E(2)$ (i.e. the set of matrices $B$ of the form $B=\left(\begin{array}{ll}e^{i \alpha} & c \\ 0 & e^{-i \alpha}\end{array}\right)$ where $\alpha$ is real and $c$ is any complex number $)$ for $\tilde{q}_{\mathrm{II}}$, and $S U(1,1)$ for $\tilde{q}_{\mathrm{III}}$. Since $\operatorname{det} \tilde{q}=\operatorname{det} \tilde{p}=a_{1}$ and $z \tilde{p} \bar{z}=\zeta \tilde{q} \bar{\zeta}=a_{2}$ there exists a Lorentz transformation connecting $p$ and $q: \tilde{p}=D \tilde{q} D^{*}$ $(D \in S L(2, C))$. Indeed, for $a_{1}<0$ that is a consequence just of the equality $p^{2}=q^{2}=a_{1}(<0)$, and for $a_{1} \geqq 0$ we have to use in addition that $\operatorname{sgn} p^{0}=\operatorname{sgn} q^{0}=\operatorname{sgn} a_{2}$. Substituting $p$ in the equality $z \tilde{p} \bar{z}=\zeta \tilde{q} \bar{\zeta}$ by $D \tilde{q} D^{*}$ we obtain

$$
z D \tilde{q} \overline{z D}=\zeta \tilde{q} \bar{\zeta}=a_{2} .
$$

Applying the statement in the beginning of the proof to (D.6) (by putting $\left.\zeta^{\prime}=z D\right)$ we find that there exists a $B \in S L(2, C)$ such that $B \tilde{q} B^{*}=\tilde{q}$ and $\zeta=z D B$. Now it is clear that the transformation $A=D B \in S L(2, C)$ satisfies (D.3) and, hence, the set $\mathfrak{M}\left(a_{1}, a_{2}(\neq 0)\right)=\left\{(p, z) \in \tilde{R}_{4} \times \tilde{C}_{2} \mid \varphi_{j}=a_{j}\right.$, $j=1,2\}$ is a homogeneous space.

On the other hand if $a_{2}=0$ (which is possible only for $p^{2} \leqq 0$ because

$$
\xi_{\mu}(z)=z \sigma_{\mu} \bar{z}
$$

is a light-like vector) the invariants (D.4) do not form a complete set. The above proof does not work in that case because the group which lives the 
equation

$$
\zeta \tilde{q} \bar{\zeta}=0
$$

(with fixed $\tilde{q}$ ) invariant is larger than the corresponding stability group of $\tilde{q}$. (It consists of all $V$ for which $V \tilde{q} V^{*}=\lambda \tilde{q}$ where $\lambda=\lambda(V)$ is an arbitrary real number.) However, it is easy to find an additional invariant function in this singular case which completes the set of invariants (D.4). The explicit expression for this additional invariant depends on the type of the vector $p$.

For $p^{2}=a_{1}=0, p$ and $\xi(z)$ (D.7) are two real orthogonal light-like vectors, and therefore, they are collinear. The factor of proportionality is the third invariant we are looking for in this case:

$$
\varphi_{3}(p, z)=\frac{1}{p_{0}} z \sigma_{0} \bar{z}=\cdots=\frac{1}{p_{3}} z \sigma_{3} \bar{z} .
$$

For $p^{2}=a_{1}<0$ the two three-dimensional zero-length Lorentz vectors ${ }^{5}$

$$
z \tilde{p} \tau_{j}^{*} \bar{z} \text { and } \sqrt{-a_{1}} \bar{z} \varepsilon^{-1} \tau_{j}^{*} \bar{z}
$$

[transforming under the representation $[-1,2]((0,1)$ in the notation of Section 3.1) of $S L(2, C)]$ are collinear with a phase factor of proportionality. To see this we mention that the equation $z \tilde{p} \bar{z}=0$ implies

$$
z \tilde{p}=C(p, z) \bar{z} \varepsilon^{-1}
$$

which makes evident the collinearity of the vectors (D.10).

To evaluate $C$ we take the complex conjugate of (D.11) and multiply by $\varepsilon$ from the right. Using the hermiticity of $\tilde{p}$ and (4.5) we find

$$
\bar{z} \varepsilon \underset{\sim}{p}=\overline{C(p, z)} z .
$$

Multiplying (D.11) by $p$ (see (4.5)) from the right and using (D.12) and the identity $\tilde{p} \underset{\sim}{p}=a_{1} 1$ we obtain

$$
|C(p, z)|^{2}=-a_{1}
$$

(in particular we see that $C=0$ for light-like $p$ so that the 3-vectors (D.10) vanish in that case). Thus, we can write down the third invariant for $a_{1}<0, a_{2}=0$ :

$$
\varphi_{3}(p, z)=\frac{\sqrt{-a_{1}} \bar{z} \varepsilon^{-1} \tau_{j}^{*} \bar{z}}{z \tilde{p} \tau_{j}^{*} \bar{z}}=e^{i \alpha}, \quad j=1,2,3 .
$$

It is not difficult to check that the set of invariants $\left\{\varphi_{1}, \varphi_{3}\right\}$ given by (D.4) and (D.9) (resp. (D.13)) is complete on the surface $\varphi_{2}=0$. 


\section{Appendix E. Expansion of Generalized Functions of $\mathscr{D}^{\prime}([-1,1])$ in Jacobi Polynomials}

We define $\mathscr{D}([-1,1]) \equiv \mathscr{D}$ as the space of infinitely differentiable functions $v(v)$ on the closed interval $[-1,1]$ with topology defined by the countable set of semi-norms (cf. (2.25))

$$
a_{k}(v)=\sup _{-1 \leqq v \leqq 1}\left|\frac{d^{k} v(v)}{d v^{k}}\right|, \quad k=0,1, \ldots
$$

(For $a_{0}(v)$ we shall use also the notation $\|v\|$.)

Let $\left\{P_{n}^{(\alpha, \beta)}(v)\right\}_{n=0}^{\infty}$ be the set of Jacobi polynomials [17] (with $\alpha, \beta>-1)$ satisfying

$$
\begin{gathered}
\left\langle P_{n}^{(\alpha, \beta)}, P_{m}^{(\alpha, \beta)}\right\rangle \equiv \int_{-1}^{1}\left(\frac{1-v}{2}\right)^{\alpha}\left(\frac{1+v}{2}\right)^{\beta} P_{n}^{(\alpha, \beta)}(v) P_{m}^{(\alpha, \beta)}(v) d v=h_{n} \delta_{n m} \\
\left(h_{n}=\frac{\Gamma(\alpha+n+1) \Gamma(\beta+n+1)}{n !(2 n+\alpha+\beta+1) \Gamma(\alpha+\beta+n+1)}\right) .
\end{gathered}
$$

(We are actually interested only in the case when $\alpha$ and $\beta$ are non-negative integers.)

We shall prove the following theorem.

Theorem E.1. Each generalized function $g(v) \in \mathscr{D}^{\prime}$ can be expanded in a series

$$
g(v)=\sum_{n=0}^{\infty} g_{n}\left(\frac{1-v}{2}\right)^{\alpha}\left(\frac{1+v}{2}\right)^{\beta} P_{n}^{(\alpha, \beta)}(v) \equiv \sum_{n=0}^{\infty} g_{n} t_{n}(v)
$$

convergent with respect to the topology of $\mathscr{D}^{\prime}$. Here $t_{n}(v)$ is a functional on $\mathscr{D}$ defined by the integral

$$
\left(t_{n}, v\right)=\int_{-1}^{1}\left(\frac{1-v}{2}\right)^{\alpha}\left(\frac{1+v}{2}\right)^{\beta} P_{n}^{(\alpha, \beta)}(v) v(v) d v,
$$

$\left\{g_{n}\right\}$ is a sequence of complex numbers of (at most) power growth:

$$
\left|g_{n}\right| \leqq A(1+n)^{q}, \quad n=0,1,2, \ldots(A=A(g), q=q(g)) .
$$

Conversely, each sequence $\left\{g_{n}\right\}$ satisfying (E.4) defines through (E.3) a continuous functional on $\mathscr{D}$.

Proof. Let $v \in \mathscr{D}$ and let

$$
E_{n}(v)=\inf _{P_{n}}\left\|v(v)-P_{n}(v)\right\|, \quad n=0,1, \ldots
$$


where the infimum is taken over all polynomials of power $p \leqq n$. The classical Jackson theorem (see [18] or [19], Section 5.1.5) implies

$$
\sup _{n>r}\left[n^{r+1} E_{n}(v)\right] \leqq \alpha_{r} a_{r+1}(v)<\infty, \quad r=0,1, \ldots ; \quad n>r
$$

where $\alpha_{r}$ are some positive constants (depending only on $r$ ).

We shall show that the set of semi-norms

$$
b_{0}(v)=a_{0}(v)=\|v\| ; \quad b_{r}(v)=\sup _{n>r}\left[n^{r} E_{n}(v)\right], \quad r=1,2, \ldots
$$

is equivalent to the set (E.1). It is readily seen from (E.6) that the seminorms (E.7) are majorized by the semi-norms (E.1). To see that the inverse is also true it is sufficient to make use of the following theorem of Dzjadyk ${ }^{10}$ ([20], Theorem 3; see also [19], Section 6.2.3).

Let the function $v(v)$ defined on $[-1,1]$ be such that for some $r, N, \lambda$ $(r, N=0,1, \ldots, 0<\lambda<1)$ and for each $n>N$ there exists a polynomial $P_{n}(v)$ of degree $p \leqq n$ such that

$$
\left[v(v)-P_{n}(v)\right] \leqq M(v)\left[\left(\frac{1}{n} \sqrt{1-v^{2}}\right)^{r+\lambda}+\frac{1}{n^{2(r+\lambda)}}\right]
$$

where $M(v)$ is a positive constant (which does not depend on $n$ ). Then $v(v)$ has $r$ th derivative and

$$
\sup _{|v| \leqq 1}\left|v^{(r)}(v)\right| \equiv a_{r}(v) \leqq \beta_{r, N, \lambda}\|v\|+\gamma_{r, N, \lambda} M(f)
$$

where $\beta$ and $\gamma$ are suitable constants.

Let $v$ be a test function from $\mathscr{D}$ and $r$ be a natural number $(r \geqq 1)$; then, in view of (E.7)

$$
E_{n}(v) \leqq n^{-2 r-1} b_{2 r+1}(v) \text { for all } n>2 r+1 .
$$

Let $P_{n}(v)$ be the polynomial (of degree $p \leqq n$ ) of least uniform deviation from $v(v)$ on $[-1,1]$; then

$$
\left|v(v)-P_{n}(v)\right| \leqq E_{n}(v) \leqq b_{2 r+1}(v)\left[\left(\sqrt{\frac{1-v^{2}}{n}}\right)^{r+\frac{1}{2}}+\frac{1}{n^{2\left(r+\frac{1}{2}\right)}}\right] .
$$

This means that condition (E.8) of Dzjadyk theorem is verified (with $\left.N=2 r+1, \lambda=\frac{1}{2}\right)$ and hence in view of (E.9)

$$
a_{r}(v)=\beta_{r}\|v\|+\gamma_{r} b_{2 r+1}(v) .
$$

10 We have reformulated Dzjadyk's theorem in a form convenient for our applications. 
Therefore, the sets of semi-norms (E.1) and (E.7) are equivalent. Let $v_{n}$ be the Fourier coefficients of the function $v(v) \in \mathscr{D}$ :

$$
v_{n}=\left\langle P_{n}^{(\alpha, \beta)}, v\right\rangle=\int_{-1}^{1}\left(\frac{1-v}{2}\right)^{\alpha}\left(\frac{1+v}{2}\right)^{\beta} P_{n}^{(\alpha, \beta)}(v) v(v) d v .
$$

We shall need the following estimate for the Jacobi polynomials ([17])

$$
h_{n}^{-1}\left|P_{n}^{(\alpha, \beta)}(v)\right| \leqq A(1+n)^{q} \quad \text { for }|v| \leqq 1
$$

where $A$ and $q$ are constants (a similar estimate without the factor $h_{n}^{-1}$ in the left-hand side is implied by (E.11)). Taking into account that $t_{n}(v)$ in (E.3) is orthogonal to any polynomial of degree $p \leqq n-1$ and using (E.11) we find the following upper bound for $\left|v_{n}\right|$ :

$$
\begin{aligned}
\left|v_{0}\right| & \leqq \eta_{\alpha \beta}\|v\|, \\
\left|v_{n}\right| & =\left|\left\langle P_{n}^{(\alpha, \beta)}, v-P_{n-1}\right\rangle\right| \leqq \eta_{\alpha \beta} \sup _{|v| \leqq 1}\left|P_{n}^{(\alpha, \beta)}(v)\right| E_{n-1}(v) \\
& \leqq A^{\prime}(1+n)^{q^{\prime}} E_{n-1}(v)
\end{aligned}
$$

(here $P_{n-1}(v)$ is a polynomial of degree $p \leqq n-1$ of least uniform deviation from $v(v)$ on $[-1,1])$. From (E.12) and (E.6) it follows that the coefficients $v_{n}$ are decreasing (in absolute value) faster than any negative power of $n$. Hence, the series

$$
\sum_{l=0}^{\infty} h_{l}^{-1} v_{l} P_{l}^{(\alpha, \beta)}(v)
$$

is uniformly convergent to some continuous function $v_{1}(v)$; the completeness of the Jacobi polynomials [17] implies that $v_{1}=v$. This allows us to obtain an inequality in the inverse direction to (E.12):

Analogously,

$$
\begin{aligned}
E_{n}(v) & \leqq\left\|v(v)-\sum_{l=0}^{n} h_{l}^{-1} v_{l} P_{l}^{(\alpha, \beta)}(v)\right\| \\
& \leqq \sum_{l=n+1}^{\infty} h_{l}^{-1}\left|v_{l}\right|\left\|P_{l}^{(\alpha, \beta)}(v)\right\| \leqq A \sum_{l=n+1}^{\infty}\left|v_{l}\right|(1+l)^{q} .
\end{aligned}
$$

$$
b_{0}(v) \leqq A \sum_{l=0}^{\infty}\left|v_{l}\right|(1+l)^{q} .
$$

From (E.12), (E.14), and (E.15) one concludes that the set of seminorms

$$
c_{r}(v)=\sup _{n \geqq 0}\left[(1+n)^{r}\left|v_{n}\right|\right], \quad r=0,1, \ldots
$$

is equivalent to the set (E.7) and, consequently, also to the set (E.1). This implies that each (continuous) linear functional on $\mathscr{D}$ has the form

$$
(g, v)=\sum_{l=0}^{\infty} g_{l} v_{l}
$$


where $g_{n}$ is a polynomially bounded sequence, and conversely, each series of the type (E.17) defines together with (E.10) a continuous linear functional on $\mathscr{D}$. This completes the proof of Theorem E.1.

\section{References}

1. Todorov, I. T., and R. P. Zaikov: Spectral representation of the covariant two-point function and infinite-component fields with arbitrary mass spectrum, ICTP, Trieste, Internal report IC/68/50 [1968]; J. Math. Phys. 10 [1969] (to appear).

2. Gel'fand, I. M., M. I. Graev, and N. Ya. Vilenkin: Generalized functions, vol. 5. Integral geometry and representation theory. New York: Academic Press 1966. See also Appendix B to Vol. 1, Properties and operations. New York: Academic Press 1964.

3. Streater, R. F., and A. S. Wightman: PCT, spin and statistics and all that. New York: W. A. Benjamin 1964; - Jost, R.: The General theory of quantized fields. Providence, R. I.: American Mathematical Society, 1965.

4. Naimark, M. A.: Linear representations of the Lorentz group. London: Pergamon Press 1963.

5. Jaffe, A. M.: Phys. Rev. 158, 1454 [1967].

6. Methée, P.: Commen. Math. Helv. 28, 225 [1954].

7. Gårding, L.: Nuovo Cimento, Suppl. 14, 45 [1959].

9. Steinmann, O.: Helv. Phys. Acta 36, 90 [1963] [Sec. 6, p. 109]; - Steinmann O.: J. Math. Phys. 4, 583 [1963].

10. Araki, H., K. Hepp, and D. Ruelle: Helv. Phys. Acta 35, 164 [1962].

11. Kamefuchi, S., and H. Umezawa: Progr. Theor. Phys. 6, 543 [1951]; - Källén, G.: Helv. Phys. Acta 25, 417 [1952]; - Lehmann, H.: Nuovo Cimento 11, 342 [1954]; Maurin, K.: Bull. Acad. Polon. Sci., Sér. Math. Ast. Phys. 7, 461 [1959];-- Rasziller, I., et al.: Nuovo Cimento A 48, 617, 635, 645 [1967].

12. Gel'fand, I. M., R. A. Minlos, and Z. Ya. Shapiro: Representations of the rotation and Lorentz group and their applications, Ch. 2, Sec. 7. London: Pergamon Press 1963; - Vilenkin, N. Ya.: Special functions and theory of group representations, Ch. III, Sec. 3. Moscow: Nauka 1965; in Russian.

13. Maurin, K.: Bull. Acad. Polon. Sci., Sér. Math. Ast. Phys. 7, 151 [1959].

14. Weyl, H.: Ann. Math. 35, 486 [1934].

15. Araki, H.: Ann. Phys. [N. Y.] 11, 260 [1960].

16. Schwartz, L.: Méthodes mathématiques pour les sciences physiques, Ch. IV. Paris: Hermann 1961.

17. Bateman, H., and A. Erdélyi: Higher transcendental functions, Vol. 2. New York: McGraw-Hill Book Co., Inc. 1953.

18. Jackson, D.: The theory of approximation, Ch. I, Theorem III. New York: Colloquium Publications, v. 111930.

19. Timan, A. F.: Theory of approximation of functions of a real variable. Moskow: Fizmatgiz 1960, in Russian.

20. Dzjadyk, V. K.: Izv. AN SSSR, Ser. Math. 20, 623 [1956].

A. I. Oksak

Institute for High Energy Physics

Serpukhov, USSR
I. T. Todorov

Institute for Advanced Study

Princeton, N. J. 08540, USA 